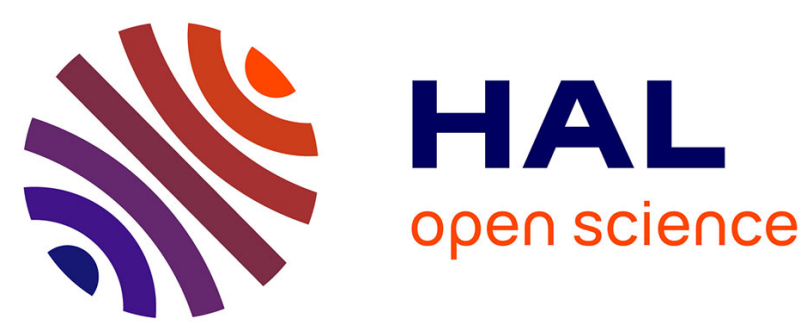

\title{
Strong effect of Penicillium roqueforti populations on volatile and metabolic compounds responsible for aromas, flavor and texture in blue cheeses
}

Thibault Caron, Mélanie Le Piver, Anne-Claire Péron, Pascale Lieben, René

Lavigne, Sammy Brunel, Daniel Roueyre, Michel Place, Pascal Bonnarme, Tatiana E Giraud, et al.

\section{To cite this version:}

Thibault Caron, Mélanie Le Piver, Anne-Claire Péron, Pascale Lieben, René Lavigne, et al.. Strong effect of Penicillium roqueforti populations on volatile and metabolic compounds responsible for aromas, flavor and texture in blue cheeses. International Journal of Food Microbiology, 2021, 354, pp.109174. 10.1016/j.ijfoodmicro.2021.109174 . hal-03202918

\section{HAL Id: hal-03202918 \\ https://hal.science/hal-03202918}

Submitted on 20 Apr 2021

HAL is a multi-disciplinary open access archive for the deposit and dissemination of scientific research documents, whether they are published or not. The documents may come from teaching and research institutions in France or abroad, or from public or private research centers.
L'archive ouverte pluridisciplinaire HAL, est destinée au dépôt et à la diffusion de documents scientifiques de niveau recherche, publiés ou non, émanant des établissements d'enseignement et de recherche français ou étrangers, des laboratoires publics ou privés. 
1 Title: Strong effect of Penicillium roqueforti populations on volatile and metabolic 2 compounds responsible for aromas, flavor and texture in blue cheeses

8

\section{9}

Running title: Impact of mold population on blue cheeses

\section{Authors:}

Thibault CARON ${ }^{1,4}$, Mélanie LE PIVER ${ }^{4}$, Anne-Claire PÉRON ${ }^{3}$, Pascale LIEBEN $^{3}$, René LAVIGNE $^{2}$, Sammy BRUNEL ${ }^{4}$, Daniel ROUEYRE ${ }^{4}$, Michel PLACE ${ }^{4}$, Pascal BONNARME $^{3}$, Tatiana GIRAUD ${ }^{1}$, Antoine BRANCA ${ }^{1}$, Sophie LANDAUD ${ }^{3}$, Christophe CHASSARD ${ }^{2 *}$

1: Ecologie Systematique Evolution, Université Paris Saclay, CNRS, AgroParisTech, 91400 Orsay, France

2: Université Clermont Auvergne, INRAE, Vetagro Sup, UMRF, 20 Côte de Reyne, 15000 Aurillac, France

3: Université Paris-Saclay, INRAE, AgroParisTech, UMR SayFood, 78850 ThivervalGrignon, France

4: Laboratoire Interprofessionnel de Production - SAS L.I.P., 34 rue de Salers, 15000

Aurillac, France

*joint senior authors

Contact information: Thibault Caron thibaultcaron864@gmail.com

Keywords: Roquefort cheese, fungi, Penicillium, domestication, volatile compounds

Acknowledgments: We thank Béatrice DESSERRE, Céline DELBES and Cécile CALLON for advice and technical assistance in microbiology, Sébastien THEIL for technical support for metabarcoding analyses, Patricia LE THUAUT, Manon SURIN and Brigitte POLLET for technical support for metabolomic analyses, Sara PARISOT for milk delivery and quality, Pierre CONCHON for technical support for image analysis, LIAL-MC for the various reference measurements in physicochemistry, Christophe LACROIX and Alfonso DIE for the determination of short-chain fatty acids in fermentation supernatants.

This study was funded by the LIP SAS, ANRT (Association Nationale Recherche Technologie), the ERC Genomefun 309403 starting grant, the BLUE ERC Proof-of-Concept grant, the Fondation Louis D grant (French Academy of Sciences) and the ANR-19-CE200002-02 Fungadapt ANR grant. 


\section{Abstract}

40 Studies of food microorganism domestication can provide important insight into adaptation

41 mechanisms and lead to commercial applications. Penicillium roqueforti is a fungus with four

42 genetically differentiated populations, two of which were independently domesticated for

43 blue cheese-making, with the other two populations thriving in other environments. Most

44 blue cheeses are made with strains from a single $P$. roqueforti population, whereas Roquefort

45 cheeses are inoculated with strains from a second population. We made blue cheeses in

46 accordance with the production specifications for Roquefort-type cheeses, inoculating each

47 cheese with a single $P$. roqueforti strain, using a total of three strains from each of the four

48 populations. We investigated differences between the cheeses made with the strains from the

49 four $P$. roqueforti populations, in terms of the induced flora, the proportion of blue color,

50 water activity and the identity and abundance of aqueous and organic metabolites as proxies

51 for proteolysis and lipolysis as well as volatile compounds responsible for flavor and aroma.

52 We found that the population-of-origin of the $P$. roqueforti strains used for inoculation had a

53 minor impact on bacterial diversity and no effect on the abundance of the main

54 microorganism. The cheeses produced with $P$. roqueforti strains from cheese populations had

55 a higher percentage of blue area and a higher abundance of the volatile compounds typical of

56 blue cheeses, such as methyl ketones and secondary alcohols. In particular, the Roquefort

57 strains produced higher amounts of these aromatic compounds, partly due to more efficient

58 proteolysis and lipolysis. The Roquefort strains also led to cheeses with a lower water

59 availability, an important feature for preventing spoilage in blue cheeses, which is subject to

60 controls for the sale of Roquefort cheese. The typical appearance and flavors of blue cheeses

61 thus result from human selection on $P$. roqueforti, leading to the acquisition of specific

62 features by the two cheese populations. These findings have important implications for our

63 understanding of adaptation and domestication, and for cheese improvement. 
64 Domestication is an evolutionary process that has been studied by many biologists since

65 Darwin. Indeed, domestication is an excellent model for understanding adaptation, because it

66 results from strong and recent selection on traits that are often known and of interest to

67 humans (Larson et al., 2014). Furthermore, studies of domestication frequently have

68 important implications for the improvement of cultivated organisms. Domesticated fungi

69 have been less studied than crops in this respect, despite being excellent models in this field

70 (Giraud et al., 2017; Gladieux et al., 2014). Most fungi can be cultured in Petri dishes, can

71 remain alive for decades when stored in freezers and are propagated asexually. All these

72 features facilitate experimentation. Fungal metabolism results in the production of various

73 compounds of interest, including fuel alcohols, enzymes and antibiotics (Bigelis, 2001). The

74 most ancient and frequent human use of fungi is for fermentation, to preserve and mature

75 food. For example, the yeast Saccharomyces cerevisiae is used in the production of bread,

76 wine and beer, and the filamentous fungus Aspergillus oryzae is used for the production of

77 soy sauce and sake (Dupont et al., 2017). These models have provided important insight into

78 mechanisms of adaptation and domestication (Almeida et al., 2014; Baker et al., 2015;

79 Gallone et al., 2016; Gibbons et al., 2012; Gonçalves et al., 2016; Libkind et al., 2011; Sicard

80 et al., 2011).

82 The Penicillium genus contains more than 300 species, several of which are used by humans.

83 For example, penicillin was discovered in $P$. rubens, and two other species, $P$. nalgiovense

84 and P. salami, are used in the production of dry-cured meat (Fleming, 1929; Ludemann et al.,

85 2010, Perrone et al., 2015). For centuries, Penicillium roqueforti (Thom) has been used in the

86 maturation of all blue cheeses worldwide (Coton et al., 2020a; Labbe et al., 2004, 2009;

87 Vabre, 2015). This fungus generates cheeses with a blue-veined appearance, by producing

88 melanized conidia within cavities in the cheese in which oxygen is readily available (Moreau, 
89 1980). Penicillium roqueforti is also found in non-cheese environments (Pitt et al., 2009;

90 Ropars et al., 2012), and four genetically differentiated clusters of individuals (i.e.,

91 populations) have been identified in $P$. roqueforti. Two populations are used for

92 cheesemaking, whereas the other two populations thrive in silage, lumber or spoiled food

93 (Dumas et al., 2020; Gillot et al., 2015; Ropars et al., 2014). Genomic and experimental

94 approaches have provided compelling evidence for the domestication of cheese P. roqueforti

95 populations (Cheeseman et al., 2014; Dumas et al., 2020; Gillot et al., 2015, 2017; Ropars et

96 al., 2015, 2016b, 2017) which have been reviewed (Coton et al., 2020b; Ropars et al.,

97 2020a). Indeed, the populations of $P$. roqueforti used to make blue cheeses display the

98 characteristic features of domesticated organisms: genetic and phenotypic differences relative

99 to non-cheese populations, with, in particular, traits beneficial for cheese production, such as

100 faster growth on cheese medium (Dumas et al., 2020; Gillot et al., 2015; Ropars et al., 2014,

101 2016), but also lower fertility and lower fitness in nutrient-poor conditions (Ropars et al.,

102 2015, 2016). Both cheese populations have lower levels of genetic diversity than the two non-

103 cheese populations, indicating an occurrence of bottlenecks (Dumas et al., 2020), which

104 typically occur during domestication. The two cheese populations are genetically and

105 phenotypically differentiated from each other, suggesting that they result from independent

106 domestication events (Dumas et al., 2020). One of the cheese populations, the non-Roquefort

107 population, is a clonal lineage with a very low level of genetic diversity used to produce most

108 types of blue cheeses worldwide. The second cheese population, the Roquefort population, is

109 genetically more diverse and contains all the strains used to produce blue cheeses from the

110 emblematic Roquefort protected designation of origin (PDO) (Dumas et al., 2020). In vitro

111 tests have shown that the non-Roquefort population displays faster tributyrin degradation (i.e.

112 a certain type of lipolysis) and higher salt tolerance, faster in vitro growth on cheese medium,

113 better exclusion of competitors than the Roquefort population (Dumas et al., 2020; Ropars et 
$114 a l ., 2014,2015)$, and an absence of toxic mycophenolic acid production (Gillot et al., 2017).

115 The specific features of the Roquefort population may result from the constraints of the PDO,

116 requiring the use of local strains and at least 90 days of maturation, and preventing the use of

117 strains from the non-Roquefort population better suited to modern modes of production

118 (Dumas et al., 2020). Genomic footprints of domestication (i.e., of adaptive genetic changes)

119 have been identified in the two $P$. roqueforti populations used for cheesemaking. Indeed, it

120 has been suggested that horizontally transferred genes found only in the non-Roquefort

121 population are involved in the production of an antifungal peptide and in lactose catabolism

122 (Cheeseman et al., 2014; Ropars et al., 2014, 2015). The effects of positive selection have

123 been detected in genes with predicted functions in flavor compound production, in each of

124 the cheese populations (Dumas et al., 2020).

125

126 Thus, the four $P$. roqueforti populations probably harbor multiple specific traits, leading to

127 the generation of cheeses with different physicochemical properties and flavors, although this

128 has yet to be tested. Assessments of the effect of the population-of-origin of the P. roqueforti

129 strain used on the features of the cheese will i) provide important fundamental knowledge

130 about the trait under selection for cheesemaking and adaptation to the cheese environment, ii)

131 provide a basis for the elucidation of other genomic changes and iii) be crucial to

132 improvements in strain use and improvement. Penicillium roqueforti is used as a secondary

133 starter for flavor production, mostly through proteolysis (i.e. casein catabolism) and lipolysis

134 during ripening (Moreau, 1980). The main characteristic feature of blue cheeses, and of

135 Roquefort PDO cheeses in particular, is their intense, spicy flavors (Kinsella et al., 1976;

136 Rothe et al., 1982). The specific volatile and metabolic compounds responsible for these

137 flavors are generated principally by lipolysis in blue cheeses (Cerning et al., 1987; Collins et 138 al., 2003) and their intensity varies among P. roqueforti strains (Dumas et al., 2020; Gillot et 
139

140

141

142

143

144

145

146

147

148

149

150

151

152

153

154

155

156

157 The differences between $P$. roqueforti populations have, to date, been studied only in vitro or

158 in very rudimentary cheese models. Here, our objective was to assess the effect of the $P$.

159

160

161

162

163

al., 2017; Larsen et al., 1999). The fatty acids released by lipolysis are the precursors of aldehydes, alcohols, acids, lactones and methyl ketones, which provide the moldy aromas typical of blue cheeses (Collins et al., 2003). Penicillium roqueforti degrades most proteins, but the efficiency of proteolysis differs between strains (Cerning et al., 1987; Dumas et al., 2020; Gillot et al., 2017; Larsen et al., 1998). The resulting peptides contribute to flavors, and their degradation into amino acids further influences cheese aroma and the growth of other microorganisms (McSweeney et al., 2000; Williams et al., 2004). Penicillium roqueforti also contributes to lactate degradation, which is necessary for deacidification and promotes the development of less acid-tolerant microorganisms (McSweeney et al., 2017). Through these effects, and by producing secondary metabolites with antimicrobial properties, P. roqueforti may also affect the microbial composition of the cheese (Kopp et al., 1979; Vallone et al., 2014). Another parameter potentially affected by $P$. roqueforti populations and restricting the occurrence of spoiler microorganisms is the lack of free water, (i.e., low water activity, Aw), which is subject to strong controls for the sale of Roquefort cheese and is affected by the degree of proteolysis (Ardö et al., 2017). The P. roqueforti population may, thus, also have an indirect effect on the features of the cheese, through various effects on beneficial or undesirable contaminants. roqueforti population-of-origin of the strain used for inoculation on the features of blue cheeses. We focused on several features considered important for cheese quality. Given the evidence from previous studies that cheese $P$. roqueforti populations have been domesticated, any differences between the cheeses produced with cheese and non-cheese populations, and/or between the two cheese populations can probably be considered to reflect selection by 
164 humans for the production of good cheeses, either on standing variation in the ancestral $P$. 165 roqueforti population or for de novo mutations. We therefore produced blue cheeses in 166 conditions very similar to those used in industrial Roquefort PDO production, using, in 167 particular, milk from the local "Lacaune" breed, with strains from the four $P$. roqueforti 168 populations. We compared several important cheese features between the four populations: i) 169 physicochemical features, relating to texture and biochemical composition, ii) cheese 170 microbiota composition and abundance, which may have effects on several cheese features, 171 iii) the blue area as a proportion of cheese slices, which is important for the blue-veined appearance of the cheese and is dependent on the growth and sporulation of $P$. roqueforti in cheese cavities, and iv) the types and abundance of the metabolic and volatile compounds

174 produced, which influence flavor and aroma. We investigated the differences in these features

175 between the cheeses produced with strains from the four $P$. roqueforti populations (Roquefort 176 cheese, non-Roquefort cheese, silage and lumber/food spoiler populations). We also 177 investigated the possible differences between cheeses made with cheese and non-cheese 178 populations, and between the Roquefort and non-Roquefort cheese populations. Assessments 179 of the traits differing between cheese and non-cheese P. roqueforti populations, and between 180 the two cheese populations, and investigations of whether the cheese populations are more 181 suitable for cheese-making, are of fundamental importance for understanding the 182 domestication of cheese fungi, through the identification of traits subjected to selection.

183 Furthermore, the identification of differences between $P$. roqueforti populations of relevance 184 for cheese making has many applied consequences for the cheese industry, in terms of strain 185 choice for different kinds of blue cheeses, paving the way for the improvement of mold 186 strains by generating progenies from crosses of the two cheese populations, and for the 187 choice of traits for measurement and selection in offspring. 
189 Materials and Methods

190 The Materials and Methods are described in more details in the Supplementary Methods.

191

192 Experimental design and fungal strains: We made cheeses with three different strains from

193 each of the four $P$. roqueforti populations (Figure 1, a strain is defined here as a haploid

194 individual obtained by monospore isolation), according to population assignments obtained in

195 a previous study based on molecular markers (Dumas et al., 2020). For cheese production

196 (Figure 1B), we used a single strain per cheese for inoculation, using a total of three different

197 strains from each of the four P. roqueforti populations (Figure 1A). Due to the limited

198 production capacity of the experimental facility, we were unable to make all the cheeses at

199 the same time. We therefore split production into three assays, each including one strain from

200 each of the four populations (Figure 1A). For each strain in each assay, we created three

201 production replicates, with two cheeses per strain in each replicate, to ensure the production

202 of sufficient material for sampling. In total, we produced 72 cheeses ( 4 strains $* 2$ cheeses $* 3$

203 replicates $* 3$ assays, Figure 1 A). The assays were performed sequentially from February to

204 April. The effect of the seasonal change in milk composition was therefore confounded with

205 the strain effect within the population, hereafter referred to as the "assay effect". The three

206 replicates within each assay were also set up at different times, a few days apart, and thus

207 with different batches of raw milk (Figure 1A). LCP (Laboratoire de Cryptogamie de Paris)

208 strains were obtained from the Muséum d'Histoire Naturelle de Paris, France, and BRFM

209 (Biological Resource Fungi Marseille') strains were obtained from the Centre International

210 de Ressources Microbiennes de Marseille, France.

211 Cheesemaking: The cheesemaking protocol was typical of the procedures used by the main

212 producers of Roquefort cheese and complied with the Roquefort PDO specifications, except

213 that the ripening process took place in artificial cellars in the INRA facilities at Aurillac and 
214 with strains from different $P$. roqueforti populations. Briefly, we slowly mixed about $35 \mathrm{~L}$ of

215 milk in each vat, and heated it to $32.5^{\circ} \mathrm{C}$. We then added $20 \mathrm{mg} \cdot \mathrm{L}^{-1}$ mesophilic starter culture

216 containing Leuconostoc spp., Lactococcus lactis subsp. cremoris, L. lactis subsp. lactis, and

217 L. lactis subsp. lactis biovar diacetylactis (FD-DVS CHN-11, CHR HANSEN, Saint-

218 Germain-lès-Arpajon, France), $25 \mathrm{mg} \cdot \mathrm{L}^{-1}$ gas-producing Leuconostoc mesenterö̈des subsp.

219 mesenteroïdes (CHOOZIT LM 57 LYO 20 DCU, DANISCO, Paris, France) and about 3.6 x

$22010^{6}$ CFU.L $L^{-1}$ P. roqueforti spores (SAS LIP, Aurillac, France). The mixture was homogenized

221 for five minutes, and we then added $0.25 \mathrm{ml}$ of active chymosin at a concentration of 520

222 mg.L $\mathrm{L}^{-1}$ (Laboratoires Humeau, La Chapelle-sur-Erdre, France). The resulting curd was cut

223 into $1.5 \mathrm{~cm}^{3}$ cubes, left to rest for five minutes, and allowed to ferment for 50 minutes. The

224 curd was drained and placed in perforated cylinders of $20 \times 10 \mathrm{~cm}$ at $26^{\circ} \mathrm{C} / 85 \%$ humidity. The

225 cheeses were turned five times after molding. On the second day, the cheeses were unmolded.

226 On the third day, the cheeses were rubbed with sterilized coarse salt and transferred to

227 ripening cellars at $11^{\circ} \mathrm{C} / 95 \%$ humidity. On the fifth day, the cheeses were resalted in the

228 same way. On the seventh day, the cheeses were pricked. They were left to ripen until day 20,

229 when they were wrapped in sterile aluminum foil and allowed to mature at $-2^{\circ} \mathrm{C}$ until day 230180.

231

232 Cheese samples were collected on days 0, 9, 20, 90 and 180. These time points are referred to 233 hereafter as "stages". Day 0 corresponded to raw milk for microbiology analysis and sowed 234 curd for metabolic and volatile compound analyses. Days 9 and 20 corresponded to half-way 235 through and the end of the ripening period, respectively. Days 90 and 180 (i.e. 3 and 6 236 months) corresponded to the minimum maturation times for the Roquefort PDO and a typical 237 maturation period for sale, respectively. 
238 Microbial and metabarcoding analyses: We estimated the concentrations of various

239 microbial communities in the initial unpasteurized milk and at various stages of cheese

240 maturation, to determine whether the $P$. roqueforti population affected the cheese microbiota

241 (for more information, see the Supplementary Methods). We counted the total number of

242 aerobic mesophilic bacteria on plate count agar (PCA; Nelson, 1940). Mesophilic lactic acid

243 bacteria were counted on Man Rogosa Sharpe agar (MRS; De Man et al., 1960) and

244 thermophilic lactic acid bacteria were counted on M17 agar (Terzaghi, 1975). Dextran-

245 positive Leuconostoc spp. were counted on Mayeux, Sandine and Elliker medium (MSE;

246 Mayeux et al., 1962). Molds and yeasts were counted on oxytetracycline gelose agar medium

247 (OGA; Mossel et al., 1970). Gram-positive catalase-positive bacteria were counted on

248 cheese-ripening bacterial medium (CRBM; Denis et al., 2001). Enterobacteria were counted

249 on violet red bile glucose (VRBG; Mossel et al., 1978). We further investigated the possible

250 effects of the $P$. roqueforti population on the identity and relative abundance of other

251 microorganisms in cheeses, by performing metabarcoding analyses on our experimental

252 cheeses at 9 and 20 days (during the ripening period), by extracting DNA, and amplifying

253 and sequencing the V3-V4 region of the $16 \mathrm{~S}$ rDNA gene. Amplicons were sequenced with

254 Illumina Miseq technology. The mean read depth of the 71 samples (36 at 9 and 20 days of

255 ripening minus one lost) was 28539 reads. Amplicon data from high-throughput sequencing

256 were analyzed with Find Rapidly OTUs in Galaxy Solution (FROGS) v3.0 (Escudié et al.,

257 2018). For each OTU, taxonomic assignment was determined with the Silva-132

258 (https://www.arb-silva.de/) and 16S rDNA RefSeq databases

259 (https://blast.ncbi.nlm.nih.gov/Blast.cgi). Four widely used diversity parameters were

260 calculated from OTU compositions: two for alpha diversity (Shannon index, Simpson index)

261 and the Bray-Curtis dissimilarity for beta diversity. 
262 Blue area: We estimated the percentage area of the cheese that was blue, on fresh inner 263 cheese slices (Figure 3B). This percentage is dependent on the formation of cavities within 264 the cheese, the growth of $P$. roqueforti within the cavities and its sporulation, as the blue 265 color is due to the melanin present in $P$. roqueforti spores. We cut all the $20-, 90-$ and $180-$ 266 day cheeses in half and took three photographs of each fresh slice with a Canon PowerShot 267 SX410 IS (JPEG format, 5152 x 3864 pixels, 100 ISO, without flash). We analyzed these 268 images with imageJ 1.52n (Schneider et al., 2012; Figure S2): (i) the brightness and contrast 269 of the raw images were standardized with a stack contrast adjustment plugin, using a 270 reference image (Figure S2A,B) (ii) rectangular selection was used to crop the images 271 manually to ensure that they contained only the cheese slices (Figure S2C), (iii) the red 272 channel was segmented with a grayscale threshold of 102 to distinguish colonized cavities 273 from the inner white parts of the cheese and empty cavities (Figure S2D), (iv) the number of 274 dark pixels in the cavities was counted and divided by the total number of pixels for the entire 275 cheese slice, to determine the percentage blue area of the slice. Within the dark patches in the 276 cheese cavities colonized by the fungus, all pixels were considered dark (script at 277 https://gitlab.com/snippets/1945218).

278 Physicochemistry: We performed standard physicochemical measurements on the cheeses.

279 We measured dry matter content, fat content as a proportion of dry matter, the moisture 280 content of the defatted cheese, total, soluble and non-protein nitrogen contents, chloride and 281 salt content, water activity and $\mathrm{pH}$ at various stages of maturation, according to reference 282 methods (for more information, see the Supplementary Methods). We measured glucose, 283 lactose, lactate, acetate and butyrate concentrations in the cheeses on days 9 and 20, by high284 performance liquid chromatography (HPLC, for more information, see the Supplementary 285 Methods). 
286 Metabolic and volatile compounds: We investigated possible differences in proteolytic and 287 lipolytic activities between the four populations, by UHPLC-MS after two extraction 288 procedures (in water and an organic solvent). We analyzed the abundance of free fatty acids 289 and residual glycerides in 90-day cheeses, by coupling a global extraction (accelerated 290 solvent extraction with hexane-isobutanol) with UHPLC-MS analysis in the positive 291 (triglycerides) and negative (fatty acids) ionization modes (for more information, see the 292 Supplementary Methods). We investigated the identity and abundance of volatile flavor and 293 aroma compounds, using a dynamic headspace system (DHS) with a Gerstel MPS 294 autosampler (Mülheim an der Ruhr, Germany) and gas chromatography-mass spectrometry 295 analysis with an Agilent 7890B GC system coupled to an Agilent 5977B quadrupole mass 296 spectrometer (Santa Clara, United States). Statistical analyses were performed with R 297 software (http://www.r-project.org/). Further details about the materials and methods are 298 provided in the Supplementary Methods.

\section{Results} investigated whether the population-of-origin of the $P$. roqueforti strains used for inoculation 304 affected the composition of the cheese microbiota, by estimating the densities of key 305 microbial communities through colony counts (CFU/g) on various specific culture media and 306 a metabarcoding approach based on $16 \mathrm{~S}$ sequencing targeting the bacteria in cheeses at 307 several stages of maturation. Based on microbial counts on plate, we found no significant 308 effect of $P$. roqueforti population on the abundance of any of the counted microorganisms, 309 including molds (i.e. $P$. roqueforti), at any sampled stage of maturation (Supplementary 310 Figure 1; Supplementary Table 1A). 
311 The metabarcoding approach targeting bacteria identified mostly sequences from the

312 Lactococcus and Leuconostoc spp. starters (about 58 and 40\%, respectively, Figure 2A,

313 Supplementary Table 1B), which are responsible for acidification and cavity formation,

314 respectively, in the cheese. The remaining sequences corresponded to 12 bacterial genera

315 frequently found in raw milk cheeses, such as Lactobacillus, Staphylococcus and

316 Arthrobacter $(<1 \%$, Supplementary Table 1B). However, the large predominance of starters

317 made it impossible to obtain sufficient data for other bacteria to assess differences in the

318 abundance of particular bacteria between cheeses made with strains from the four $P$.

319 roqueforti populations (Supplementary Table 1C). We also targeted eukaryotes, based on ITS

320 sequences, in a few samples. Most sequences were assigned to P. roqueforti spp. (89\%) and,

321 to a lesser extent, Candida spp. (10\%, data not shown), so no further analyses were

322 performed on the remaining low-abundance sequences. We estimated three OTU (operational

323 taxonomic unit) diversity parameters based on bacterial barcode sequence abundances, to

324 measure OTU richness and/or evenness. Bray-Curtis dissimilarity showed that cheeses made

325 with strains from the same $P$. roqueforti population were no more similar than those made

326 with strains from different $P$. roqueforti populations. However, we found a significant effect

327 of $P$. roqueforti population, in addition to a stage effect, on the Shannon and Simpson

328 diversity indices (Figures $2 \mathrm{~B}$ and $2 \mathrm{C}$ ). Cheeses made with strains from the cheese $P$.

329 roqueforti populations tended to have a higher bacterial OTU diversity, particularly at nine

330 days of maturation and for the Roquefort population (Figures $2 \mathrm{~B}$ and $2 \mathrm{C}$ ), although the post-

331 hoc analyses were not powerful enough to detect significant pairwise differences

332 (Supplementary Table 1C). There may also be undetected differences at species level or for

333 low-abundance microorganisms that might nevertheless have substantial effects. However,

334 even if this were the case, it would constitute an indirect effect of the $P$. roqueforti

335 population, as this was the only difference during our cheesemaking process. 
337 Higher proportion of blue area in cheeses produced with cheese $P$. roqueforti

338 populations. The percentage blue area in cheese cavities was determined on freshly cut

339 cheese slices (Figure 3B). The blue veins result from the formation of cavities in the cheese,

340 and the growth and sporulation of $P$. roqueforti in these cavities. The percentage blue area

341 was significantly higher in cheeses produced with cheese population strains than in those

342 produced with non-cheese population strains (Figure 3A and 3B; Supplementary Table 1D).

343 We also found a significant decrease in the percentage blue area from 20 to 180 days of

344 maturation, for all populations except the Roquefort population, for which the percentage

345 blue area remained relatively constant (Figure 3A; Supplementary Table 1D).

347 More efficient proteolysis and lipolysis by the Roquefort $\boldsymbol{P}$. roqueforti population. We

348 investigated the proteolysis and lipolysis efficiencies of the four populations in cheeses after

34990 days of maturation. Both targeted and non-targeted chromatographic analyses showed that

350 proteolytic efficiency was highest in the Roquefort $P$. roqueforti population. We performed

351 the targeted analysis with standards for the principal 23 amino acids (Supplementary Table

352 2A). We found that eight amino acids discriminated significantly between cheeses made with

353 the different P. roqueforti populations (Supplementary Table 1E, mainly ornithine, leucine,

354 and alanine), 15 discriminated between the cheese and non-cheese populations (mainly

355 spermidine, isoleucine, methionine, glutamic acid, citrulline, serine and leucine) and 14

356 distinguished between the Roquefort and non-Roquefort populations (Supplementary Figure

357 3A, mainly valine, leucine, isoleucine, serine and threonine). The cheeses made with strains

358 from cheese populations, and from the Roquefort population, in particular, had a higher total

359 amino-acid concentration (Supplementary Tables 1E and 2B). 
360 We also assessed proteolytic activity in a non-targeted analysis (fingerprint approach) on

361 whole chromatograms (8,364 signals), which provided much more powerful discrimination significantly different from each other, and proteolysis was least efficient for the silage population (Figure 4; Supplementary Table 1F).

369 Lipolysis was also more efficient for the Roquefort population than for the other populations.

370 We investigated whether the $P$. roqueforti population influenced the abundance of free fatty

371 acids and residual glycerides, as a proxy for the efficiency of lipolysis, in 90-day cheeses,

372 with targeted and non-targeted chromatographic analyses in the positive and negative

373 ionization modes. We specifically targeted glycerides and free fatty acids. In the targeted

374 analysis, we identified seven free fatty acids and 20 triglycerides, and found that three free

375 fatty acids (stearic, oleic and linoleic acids) were significantly more concentrated in cheeses

376 made with Roquefort strains than in those made with strains from non-Roquefort populations

377 (Supplementary Table 1G). In the non-targeted analysis, we obtained 3,094 signals and

378 observed a higher abundance for organic signals specific to free fatty acids, indicating the

379 most efficient lipolysis, in cheeses made with strains from the Roquefort population, followed

380 by the lumber and non-Roquefort cheese populations, which were very similar to each other,

381 with lipolytic efficiency lowest for cheeses made with strains from the silage population

382 (Figure 5; Supplementary Table 1H). For residual glycerides, we obtained 8,472 signals, with

383 no significant difference between the populations (Supplementary Figure 4; Supplementary

384 Table 1I). 
386 We performed the most relevant standard physicochemical measurements on raw milk and on

387 the cheeses at 9, 20, 90 and 180 days of maturation. As expected, we observed a maturation 388 stage effect for 11 of the 16 physicochemical parameters (Supplementary Table 1J). Non389 protein nitrogenous content was significantly higher in cheeses inoculated with strains from 390 cheese $P$. roqueforti populations than in cheeses inoculated with strains from the other 391 populations, consistent with the greater efficiency of proteolysis associated with these strains 392 (Supplementary Figure 5A). Cheese water activity differed significantly between the cheeses made with strains from the four $P$. roqueforti populations (Supplementary figure 5B): it was significantly lower for the Roquefort cheese population than for the non-Roquefort cheese and silage populations (see statistics in the Supplementary Table 1J).

397 Strong influence of $\boldsymbol{P}$. roqueforti population on volatile compound production. We 398 investigated the effect of $P$. roqueforti population on cheese volatile compounds in 90-day 399 cheeses. We focused on the GC-MS data for the 40 principal volatile compounds considered 400 to be most important for the aromatic quality of blue cheeses (Rothe et al., 1982): 11 acids, 40112 ketones, 10 esters, six alcohols and one aldehyde (Supplementary Table 3). We found that 402 P. roqueforti population strongly influenced the relative abundance of the compounds from 403 these aromatic families in the cheeses (Supplementary Table 1K; Figures 6 and 7, and see 404 below). In fact, the odors of the cheeses differed considerably (pers. obs.): the cheeses made 405 with strains from the cheese $P$. roqueforti populations smelled as good as typical ripened blue 406 cheeses, whereas those made with strains from non-cheese $P$. roqueforti populations had 407 unpleasant odors, similar to those of a wet mop (Supplementary Figure 6; personal 408 observation). 
410 The abundance of acids, methyl ketones and secondary alcohols resulting from proteolysis

411 and lipolysis, and contributing to the typical flavor of blue cheese, was higher in cheeses

412 produced with strains from cheese populations than in those produced with strains from non-

413 cheese populations (Figure 6). These compounds were present at a particularly high

414 abundance in cheeses made with strains from the Roquefort population. Four of the 40

415 compounds analyzed were proteolysis by-products (primary alcohols: 3-methyl-butanal, 3-

416 methyl-butanol and isopropyl-alcohol, hereafter referred to as alcohols I, and 3-methyl-

417 butanoic acid, hereafter referred to as acid I; Supplementary Table 3). The abundance of

418 alcohols I was significantly higher in cheeses made with strains from cheese $P$. roqueforti

419 populations than in those made with strains from non-cheese populations, and the highest

420 values were obtained for the Roquefort population (mainly 3-methyl-butanol; Figure 6;

421 Supplementary Table 1K). Acid I (i.e. 3-methyl-butanoic acid) was also present in higher

422 abundance in cheeses made with strains from the Roquefort population than in other cheeses

423 (Figure 6). Two acids, by-products of glycolysis (acetic and propionic acid, hereafter referred

424 to as acids II), were present at higher abundance in cheeses made with strains from the

425 Roquefort and lumber/food spoiler P. roqueforti populations than in other cheeses (Figure 6;

426 Supplementary Tables $1 \mathrm{~K}$ and 3 ). The other 35 aromatic compounds (i.e. acids from beta-

427 oxidation, hereafter referred to as acids III, ketones, secondary alcohols hereafter referred to

428 as alcohols II, and esters) were almost all direct or indirect by-products of lipolysis

429 (Supplementary Table 3). The abundance of acids III was higher in cheeses made with strains

430 from the Roquefort and lumber/food spoiler populations than in cheeses made with strains

431 from the non-Roquefort cheese population (mainly butanoic, pentanoic, hexanoic and

432 octanoic acid; Figure 6; Supplementary Table 1K). The levels of these compounds were

433 lowest in cheeses made with strains from the silage population. Esters and methyl ketones

434 (especially 2-pentanone and 2-heptanone) were present at higher abundance in cheeses made 
435 with strains from cheese $P$. roqueforti populations (Supplementary Table 1K). Cheeses made

436 with strains from the Roquefort population contained the highest abundance of methyl

437 ketones, and these compounds were barely detectable in cheeses made from silage population

438 strains (mainly 2-heptanone, 2-pentanone and 2-nonanone; Figure 7A). The abundance of

439 alcohols II, particularly 2-heptanol, was also much higher in cheeses made with Roquefort

440 population strains than in other cheeses (Supplementary Table 1K; Figure 7B).

441

442

\section{Discussion}

444 Cheese $P$. roqueforti populations have been selected to produce better blue cheeses.

445 Measurements of multiple features of blue cheeses made under conditions resembling those

446 typically used in commercial Roquefort production revealed a strong influence of the

447 differentiated $P$. roqueforti populations on several aspects of cheese quality. The cheese

448 populations appeared the best adapted to cheesemaking, in terms of both the appearance and

449 volatile compound content of the resulting cheese. The differences between the four $P$.

450 roqueforti populations and the more appealing cheeses produced with strains from the cheese

451 populations suggest that humans have exerted selection for the production of better cheeses,

452 and this corresponds to domestication. Indeed, we found that cheese P. roqueforti strains

453 produced a higher percentage blue area on cheese slices, an important visual aspect of blue

454 cheeses. We also found that proteolysis and lipolysis were more efficient in cheeses made

455 with Roquefort population strains than in cheeses made with strains from the other $P$.

456 roqueforti populations, resulting in a higher abundance of desirable volatile compounds,

457 including alcohols and associated acids. Cheese water activity was lower in cheeses made

458 with strains from the Roquefort population, probably due to more efficient proteolysis (Ardö

459 et al., 2017). We found no significant difference in the identities and abundances of 
460 microorganisms between the cheeses made with strains from the four $P$. roqueforti 461 populations. Some minor differences in bacterial alpha diversity were observed, however, and

462 the differences in all other measurements than diversity between cheeses probably reflected a

463 direct effect of the specific features of the $P$. roqueforti population, although minor indirect

464 effects involving the induction of more diverse bacterial communities by cheese $P$. roqueforti

465 strains may also have occurred. Overall, our findings strongly support the view that cheese $P$.

466 roqueforti populations have been selected by humans for better appearance and aroma. This

467 selection may have involved the choice of the most beneficial strains for making good

468 cheeses from standing variation, and/or the selection of de novo genetic changes. Previous

469 studies found footprints of genomic changes in cheese populations in the form of beneficial

470 horizontal gene transfers and positive selection (Dumas et al., 2020; Ropars et al., 2015).

472 Previous studies reported differences between $P$. roqueforti populations, in terms of growth,

473 lipolysis and proteolysis, but on synthetic media (Dumas et al., 2020; Ropars et al., 2015).

474 Here, using experimental cheeses made in commercial cheese production conditions, we

475 reveal important features specific to cheese $P$. roqueforti populations, and to the Roquefort

476 and non-Roquefort cheese populations. These findings are important in the context of

477 domestication, for understanding rapid adaptation and diversification, and future studies

478 based on quantitative trait mapping may be able to identify further genomic changes

479 responsible for the specific features of the populations, according to the contrasting

480 phenotypes revealed here. Progenies can indeed be obtained from crosses between strains

481 from different populations of $P$. roqueforti (Ropars et al., 2015), and this could facilitate

482 strain improvement through recombination between the different populations. Our results are,

483 therefore, also important for improving blue cheese production. 
485 The four $\boldsymbol{P}$. roqueforti populations induce similar microbiotas, but water availability is 486 lower with cheese population strains, restricting the occurrence of spoiler

487 microorganisms. Based on microbiological counts, we found no significant differences in

488 abundance for any of the species monitored between cheeses made with strains from the four 489 populations of $P$. roqueforti. In particular, we found no significant difference in the 490 abundance of molds on Petri dishes. However, microbiological counts are known to provide 491 poor estimates of fungal biomass, especially for mycelium growth (Schnurer, 1993).

493 The abundance and identity of the microorganisms studied were globally similar to those in

494 four commercial Roquefort cheeses (personal information from C. Callon; Devoyod et al., 495 1968) and closely related blue cheeses (Diezhandino et al., 2015). The metabarcoding 496 approach suggested that the different $P$. roqueforti populations induced bacterial 497 communities of different levels of diversity. The cheese populations, and the Roquefort 498 population in particular, were associated with the highest level of alpha diversity. The 499 differences in cheese bacterial diversity, although minor, suggest that the differences between 500 cheeses made with strains from the four P. roqueforti populations may be due not only to a 501 direct effect of $P$. roqueforti population, but also to an indirect effect mediated by the 502 induction of bacterial communities of different diversities. The large predominance of 503 bacterial starters made it impossible to collect sufficient data for an assessment of the 504 differences in relative abundance between subdominant bacterial species on the basis of 505 metabarcoding. We also found a significant difference in water activity between cheeses 506 made with strains from different $P$. roqueforti populations, the lowest value obtained being 507 that for the Roquefort population. This may also reflect human selection, as low water 508 activity restricts the occurrence of spoiler microorganisms. This characteristic is, therefore, 509 subject to tight control in Roquefort cheeses for sale, particularly those for export. 
511 Cheese $P$. roqueforti populations produce bluer cheeses. We found significantly higher

512 percentage blue areas in cheese slices from cheeses made with cheese P. roqueforti strains

513 than in those made from non-cheese strains, potentially reflecting greater $P$. roqueforti

514 growth in cheese and/or a higher sporulation efficiency in cavities. The percentage blue area

515 in cheese slices also depends on the formation of cavities in the cheese, as P. roqueforti can

516 only sporulate in cavities in which oxygen is available. The cavities are mostly generated by

517 the gas-producing bacterium Leuconostoc mesenteroides, the abundance of which did not

518 differ between the cheeses made with strains from different $P$. roqueforti populations,

519 suggesting a direct effect of $P$. roqueforti populations on the blueness of cheese slices. The

520 significantly higher percentage blue area in slices of cheeses made with cheese P. roqueforti

521 strains than in those made with non-cheese strains therefore probably reflects better cheese

522 and cavity colonization and sporulation, probably due to selection on the basis of appearance.

523 The percentage blue area decreased by the end of maturation, perhaps due to the death of the

524 fungus. Only cheeses made with Roquefort strains retained a high percentage blue area at 90

525 days of maturation, again potentially reflecting selection in pre-industrial times, when

526 Roquefort cheeses had to be stored for several months at cave temperature before sale. The

527 minimum maturation time for Roquefort PDO remains 90 days, which is longer than for other

528 blue cheeses. These findings contrast with a previous study showing that a non-Roquefort

529 population colonized the cavities of model cheeses better than other populations (Dumas et

530 al., 2020); this discrepancy may reflect differences between studies in terms of the

531 measurements used (total percentage blue area versus percentage blue area within cavities),

532 the type of milk (ewe versus goat) or the mode of cheesemaking (rudimentary models versus

533 commercial-like cheeses). Our findings are consistent with the presence of horizontally 
534 transferred genes in cheese populations with predicted functions in fungal development,

535 including sporulation and hyphal growth (Dumas et al., 2020).

537 Proteolysis and lipolysis are more efficient in the Roquefort $P$. roqueforti population.

538 Based on chemical analyses and powerful chromatographic discrimination methods, we

539 showed that the abundance of amino acids and small peptides (i.e., residual products of

540 proteolysis) was highest in cheeses made with Roquefort $P$. roqueforti strains. Thus, these

541 strains had the highest capacity for proteolysis, which is an important process in

542 cheesemaking. Indeed, proteolysis contributes to the development of cheese texture, flavors

543 and aromas (Andersen et al., 2010; Ardö, 2006, 2017; McSweeney, 1997; Roudot-Algaron,

544 1996). Previous measurements of proteolytic activity in synthetic media detected significant

545 differences between $P$. roqueforti populations, but not between the two cheese populations

546 (Dumas et al., 2020). We show here that experimental cheeses made with strains from the

547 Roquefort population have a higher content of residual products of proteolysis, a sign of more

548 advanced ripening.

550 We also found that lipolysis was more efficient in the cheeses made with strains from the

551 Roquefort $P$. roqueforti population. By contrast, previous studies in synthetic media found

552 that lipolysis was most efficient in the non-Roquefort population (Dumas et al., 2020). The

553 discrepancy between these studies demonstrates the need for measurements in real cheeses

554 for the reliable assessment of metabolic activities. Lipolytic activity is known to affect cheese

555 texture and the production of volatile compounds affecting pungency (Alonso et al., 1987;

556 González De Llano et al., 1990, 1992; Martín et al., 2016; Thierry et al., 2017; Woo et al.,

557 1984). The more efficient proteolysis and lipolysis in the Roquefort $P$. roqueforti population 
558 should have a strong impact on cheese texture and flavor. It therefore probably results from

559 selection to obtain better cheeses, i.e. from a domestication process, as previously reported

560 for other fungi (Almeida et al., 2014; Baker et al., 2015; Gallone et al., 2016; Gibbons et al.,

561 2012; Gonçalves et al., 2016; Libkind et al., 2011; Sicard et al., 2011). Roquefort cheeses are

562 widely considered to be the blue cheeses with the strongest aromas and flavors. The less

563 efficient lipolysis and proteolysis in the non-Roquefort population may result from more

564 recent selection for milder cheeses.

565

566 Cheese $P$. roqueforti populations produce cheeses with more abundant and diverse

567 volatile compounds. We found major differences between the cheeses made with strains

568 from different $P$. roqueforti populations, in terms of the volatile compounds resulting from

569 lipolysis and, to a lesser extent, also from proteolysis. Only four of the aromatic compounds

570 detected in our cheeses (3-methyl-butanal, 3-methyl-butanol, isopropyl-alcohol and 3-methyl-

571 butanoic acid) were by-products of casein proteolysis (McSweeney et al., 2000), and the

572 concentrations of these molecules were significantly higher in cheeses made with Roquefort

573 P. roqueforti strains, consistent with the higher proteolysis efficiency and amino-acid

574 precursor (i.e. valine, leucine and isoleucine) concentrations of these strains. These

575 compounds produce fruity (banana), cheesy and alcoholic notes, which were probably

576 important selection criteria during the domestication of the Roquefort $P$. roqueforti

577 population. For the products of metabolic pathways leading from amino acids to alcohols

578 (Ehrlich pathway with aldehyde reduction) or acids (aldehyde oxidation; Ganesan et al.,

579 2017), the higher concentration of alcohols than of acids observed for all populations is

580 consistent with the general micro-aerobic conditions of blue cheese cavities.

581 Most of the aromatic compounds identified were direct or indirect by-products of lipolysis, 582 consistent with the known key role of lipolysis in the generation of typical blue cheese aroma 
583 (Cerning et al., 1987; Collins et al., 2003). The aromatic compounds resulting from lipolysis 584 belonged to four chemical families (acids, methyl ketones, secondary alcohols and esters).

585 Methyl ketones were the most diverse and abundant for cheese P. roqueforti populations, 586 particularly for the Roquefort population, in which abundance was highest for 2-pentanone 587 and 2-heptanone; 2-heptanone underlies the characteristic "blue cheese" sensory descriptor 588 (Anderson et al., 1966; González De Llano et al., 1990, 1992; Moio et al., 2000). In P. 589 roqueforti, methyl ketones with odd numbers of carbons are mostly produced by fatty-acid 590 beta-oxidation, whereas those with even numbers of carbons may be produced by the beta591 oxidation or autoxidation of fatty acids (Spinnler, 2011). These compounds are produced by 592 the decarboxylation of hexanoic acid and octanoic acid, respectively, which were the most 593 abundant acids found in our cheeses. This reaction is considered to be a form of 594 detoxification, because methyl ketones are less toxic than acids (Kinderlerer, 1993; Spinnler, 595 2011). Interestingly, this pathway appeared to be more active in the cheese $P$. roqueforti 596 populations, as methyl ketone levels were lower in cheeses made with lumber (four-fold difference) and silage (10-fold lower) strains than in cheeses made with cheese population strains. Methyl ketone concentrations were not directly associated with the concentrations of 599 their precursors (acids), the highest concentrations being found in the lumber and Roquefort 600 populations. The biosynthesis pathway producing methyl ketones must, therefore, be more 601 efficient in cheese populations, particularly the non-Roquefort population. The cheese $P$. 602 roqueforti populations were probably selected for their higher acid detoxification capacity, as 603 this produces aromatic compounds with a very positive impact on flavor (Spinnler, 2011).

604 The concentrations of secondary alcohols (resulting from the reduction of methyl ketones) 605 were also higher in cheeses produced by cheese $P$. roqueforti strains, particularly those of the 606 Roquefort population, for which they were seven times higher than for the non-Roquefort 607 cheese population and 20 times higher than for the silage/lumber populations; 2-heptanol was 
608 the major alcoholic compound produced. The reduction of 2-heptanone to 2-heptanol occurs

609 specifically in anaerobic conditions and is much stronger in the Roquefort population; aerobic

610 conditions were similar for all the populations. The Roquefort $P$. roqueforti population may

611 also have been selected for this feature, as secondary alcohols provide "fruity notes", which

612 are associated with better aromatic quality (Spinnler, 2011). Methyl ketones may be reduced

613 to alcohols by an alcohol dehydrogenase, as occurs when aldehyde is reduced to alcohol via

614 the Ehrlich pathway. Alcohol dehydrogenase genes may thus have been targets of selection in

615 the Roquefort $P$. roqueforti population, although they were not detected as evolving under

616 positive selection in a previous study (Dumas et al., 2020).

617 We also found higher levels of esters in cheeses made with cheese P. roqueforti populations.

618 Esters are produced principally by the esterification of ethanol with acids generated by beta-

619 oxidation. Leuconostoc starters can produce ethanol, and ester synthesis has also been

620 described as a detoxification mechanism (Mason et al., 2000). These results further indicate

621 that cheese $P$. roqueforti populations, particularly the Roquefort population, have been

622 selected for acid detoxification capacity, leading to a large variety of less toxic aromatic

623 compounds with strong aromas and flavors.

624 Overall, the aromas of cheeses made with cheese $P$. roqueforti strains had more appealing 625 aromas, and this was particularly true for cheeses made with Roquefort strains. These aroma 626 properties probably reflect selection by humans. The cheeses made with silage and lumber 627 populations had a mildly unpleasant smell, whereas those made with cheese strains smelled 628 like typical blue cheeses, with cheeses made with Roquefort strains having the strongest 629 smell. This may reflect previously reported horizontal gene transfers in cheese populations, 630 involving genes with predicted functions in lipolysis or amino-acid catabolism, and the 631 positive selection of genes involved in aroma production (Dumas et al., 2020). We compared 632 P. roqueforti populations between cheeses made following commercial modes of production, 
633 which represents a major advance relative to previous studies based on experimental models

634 or synthetic media (Dumas et al., 2020; Gillot et al., 2017). We used unpasteurized ewe's

635 milk, in accordance with the requirements for Roquefort PDO production, which also affects

636 cheese aromas. In future studies, it would be interesting to determine whether the use of

637 pasteurized or unpasteurized ewe's milk or cow's milk leads to similar specific features of the

638 Roquefort versus non-Roquefort cheese $P$. roqueforti populations, as there may have been

639 selection during domestication, leading to an adaptation of the Roquefort population for the 640 catabolism of unpasteurized ewe's milk.

642 Conclusion. We show that the $P$. roqueforti population has a strong impact on cheese

643 quality, appearance and aroma. The populations used for cheesemaking led to bluer cheeses,

644 with better aromas, probably due to domestication involving the selection of multiple fungal

645 traits by humans seeking to make the best possible cheeses. French cheese producers have

646 been inoculating cheeses with $P$. roqueforti spores from moldy rye bread since the end of the

$64719^{\text {th }}$ century (Labbe et al., 2004, 2009; Vabre, 2015). This process made it possible for them

648 to re-inoculate with the strains producing the best cheeses, thereby applying a strong selection

649 pressure. The two cheese populations displayed a number of specific features, with the

650 Roquefort population notably producing more intense and specific aromas and flavors. The

651 selection of different fungal varieties for different usages has also been reported in the

652 fermenting yeast Saccharomyces cerevisiae (Gallone et al., 2016; Legras et al., 2018).

653 Previous studies on $P$. roqueforti detected recurrent changes in amino acids and horizontal

654 gene transfers in cheese populations, both of which facilitated rapid adaptation (Dumas et al.,

655 2020; Ropars et al., 2015). Our findings provide greater insight into P. roqueforti

656 domestication and pave the way for strain improvement through the targeting of relevant

657 traits. A protocol inducing sexual selection has been developed in $P$. roqueforti (Ropars et al., 
658 2014), making it possible to perform crosses between strains from the two cheese

659 populations, each of which harbors very little genetic diversity (Dumas et al., 2020), to

660 generate variability and to identify strains with high levels of performance. The results of this

661 study will facilitate the choice of the parental strains for crossing and of the most important

662 phenotypes to be measured in the offspring. Parental strains with strongly contrasting

663 phenotypes for the traits important for cheesemaking that we found to be differentiated

664 between populations (such as volatile compound production, lipolysis and proteolysis) should

665 be used, to maximize variability in the progeny. Other traits may also be worth investigating

666 in the future, to understand the changes that have occurred during the domestication of the

667 two cheese $P$. roqueforti populations, particularly as concerns toxin production in cheeses.

668 Previous in vitro studies have shown a lack of production of the toxic mycophenolic acid by

669 the non-Roquefort population due to a deletion in one of the genes of the synthesis pathway

670 (Gillot et al., 2017). In the P. camemberti fungus, domestication has also led to a lack of

671 production of a mycotoxin due to a deletion in a gene (Ropars et al., 2020b). 
674

675

676

677

678

679

680

681

682

683

684

685

686

687

688

689

690

691

692

693

694

695

696

697

698

699

700

701

702

703

704

705

706

707

708

709

710

\section{References}

Almeida, M., Hébert, A., Abraham, A.-L., Rasmussen, S., Monnet, C., Pons, N., DelbèsPaus, C., Loux, V., Batto, J.-M., Leonard, P., Kennedy, S., Ehrlich, S., Pop, M., Montel, M.C., Irlinger, F. \& Renault, P. (2014). Construction of a dairy microbial genome catalog opens new perspectives for the metagenomic analysis of dairy fermented products. BMC Genomics, 15,1101 .

Alonso, L., Juarez, M., Ramos, M., \& Martin $\square$ Alvarez, P. J. (1987). Effects of changes during ripening and frozen storage on the physicochemical and sensory characteristics of Cabrales cheeses. International Journal of Food Science \& Technology, 22(5), 525-534.

Andersen, L. T., Ardö, Y., \& Bredie, W. L. P. (2010). Study of taste-active compounds in the water-soluble extract of mature Cheddar cheese. International Dairy Journal, 20(8), 528536.

Andersson, A. F., Lindberg, M., Jakobsson, H., Bäckhed, F., Nyrén, P., \& Engstrand, L. (2008). Comparative analysis of human gut microbiota by barcoded pyrosequencing. PLoS ONE, 3(7), e2836.

Anderson, D. F., \& Day, E. A. (1966). Quantitation, evaluation, and effect of certain microorganisms on flavor components of blue cheese. Journal of Agricultural and Food Chemistry, 14(3), 241-245.

Ardö, Y. (2006). Flavour formation by amino acid catabolism. Biotechnology Advances, 24(2), 238-242.

Ardö, Y., McSweeney, P. L. H., Magboul, A. A. A., Upadhyay, V. K., \& Fox, P. F. (2017). Biochemistry of cheese ripening: proteolysis. In P. L. H. McSweeney, P. F. Fox, P. D. Cotter, \& D. W. Everett (Eds.), Cheese: Chemistry, Physics and Microbiology (Fourth Edi, pp. 445482). Elsevier Ltd.

Auguie, B. (2017). gridExtra: miscellaneous functions for "Grid" graphics. Retrieved from https://cran.r-project.org/web/packages/gridExtra/index.html

Baker, E. P., Wang, B., Bellora, N., Peris, D., Hulfachor, A. B., Koshalek, J. A., Libkind, D. \& Hittinger, C. T.(2015). The genome sequence of Saccharomyces eubayanus and the domestication of lager-brewing yeasts. Molecular Biology and Evolution, 32(11), 2818-2831.

Bates, D., Mächloer, M., Bolker, B. M., \& Walker, S. C. (2015). Fitting linear mixed-effects models using lme4. Journal of Statistical Software, 67(1), 1-48.

Bigelis, R. (2001). Fungal fermentation: industrial. Encyclopedia of Life Sciences, 1-8.

Bjørn-Helge, M., Wehrens, R., \& Hovde Liland, K. (2018). pls: partial least squares and principal component regression ( $\mathrm{R}$ package version 2.7-0). Retrieved from https://cran.rproject.org/package $=$ pls

Cerning, J., Gripon, J.-C., Lamberet, G., \& Lenoir, J. (1987). Les activités biochimiques des Penicillium utilisés en fromagerie. Le Lait, 67(1), 3-39. 
711 Cheese and processed cheese: determination of chloride content potentiometric titration

712 method. International standards 4a. International Dairy Federation, Brussels, Belgium. 1988.

713 https://www.iso.org/standard/43922.html. ISO 5943:2006 [IDF 88:2006]

714 Cheese and processed cheese: determination of total solids content. International standards

715 4a. (1982). International Dairy Federation, Brussels, Belgium. Brussels, Belgium.

716 http://doi.org/ISO 5534:2004 [IDF 4:2004]

717 Cheeseman, K., Ropars, J., Renault, P., Dupont, J., Gouzy, J., Branca, A., et al.. (2014).

718 Multiple recent horizontal transfers of a large genomic region in cheese making fungi. Nature

719 Communications, 5, 2876.

720 Collins, Y. F., McSweeney, P. L. H., \& Wilkinson, M. G. (2003). Lipolysis and free fatty

721 acid catabolism in cheese: a review of current knowledge. International Dairy Journal,

722 13(11), 841-866.

723

724

725

726

727

728

729

730

731

732

733

734

735

736

737

738

739

740

741

742

743

744

745

746

747

748

Coton, E., Coton, M., Hymery, N., Jany, J. L., Mounier, J., \& Jany, J. L. (2020b). Penicillium roqueforti $\square$ : an overview of its genetics, physiology, metabolism and biotechnological applications. Fungal Biology Reviews, https://doi.org/10.1016/j.fbr.2020.03.001.

Coton, E., Jany, J.-L., \& Coton, M. (2020a). Yeasts and Molds: Penicillium roqueforti. In Reference Module in Food Science (pp. 1-8). Elsevier.

De Man, J. C., Rogosa, M., \& Sharpe, M. E. (1960). A medium used for the cultivation of Lactobacilli. Journal of Applied Bacteriology, 23, 130-135.

Denis, C., Gueguen, M., Henry, E., \& Levert, D. (2001). New media for the numeration of cheese surface bacteria. Le Lait, 81(3), 365-379.

Devoyod, J. J., \& Muller, M. (1969). La flore microbienne du fromage de Roquefort III. Les streptocoques lactiques et les Leuconostoc $\square$; Influence de différents micro-organismes de contamination. Le Lait, 487, 369-399. Retrieved from https://doi.org/10.1051/lait:196948715

Diezhandino, I., Fernández, D., González, L., McSweeney, P. L. H., \& Fresno, J. M. (2015). Microbiological, physico-chemical and proteolytic changes in a Spanish blue cheese during ripening (Valdeón cheese). Food Chemistry, 168, 134-141.

Dumas, É., Feurtey, A., Rodríguez de la Vega, R. C., Le Prieur, S., Snirc, A., Coton, M., Thierry, A., Coton, E., Le Piver, M., Roueyre, D., Ropars, J., Branca, A. \& Giraud, T. (2020). Independent domestication events in the blue-cheese fungus Penicillium roqueforti. Molecular Ecology, 33913(January), 451773.

Dupont, J., Dequin, S., Giraud, T., Le Tacon, F., Masit, S., Ropars, J., Richard, F. \& Selosse, M.-A. (2017). Fungi as a source of food. Microbiology Spectrum, 5(3), 1-22.

Duval, P., Chatelard-Chauvin, C., Gayard, C., Rifa, E., Bouchard, P., Hulin, S., Picque, D. \& Montel, M.-C. (2016). Microbial dynamics in industrial blue veined cheeses in different packaging. International Dairy Journal, 56, 198-207.

Edgar, R. C., Haas, B. J., Clemente, J. C., Quince, C., \& Knight, R. (2011). UCHIME improves sensitivity and speed of chimera detection. Bioinformatics, 27(16), 2194-2200. 
749 Escudié, F., Auer, L., Bernard, M., Mariadassou, M., Cauquil, L., Vidal, K., Maman, S.,

750 Hernandez-Raquet, G., Combes, S. \& Pascal, G. (2018). FROGS: Find, Rapidly, OTUs with

751 Galaxy Solution. Bioinformatics, 34(8), 1287-1294.

752 Fleming, A. (1929). On the antibacterial action of cultures of a Penicillium, with special

753 reference to their use in the isolation of B. influenza. British Journal of Experimental

754 Pathology, 10(3), 226-236.

755 Fox, J., \& Weisberg, S. (2019). An $\{R\}$ Companion to applied Regression. (T. Oaks, Ed.)

756 (Third). Sage. Retrieved from https://socialsciences.mcmaster.ca/jfox/Books/Companion/

757 Gallone, B., Steensels, J., Prahl, T., Soriaga, L., Saels, V., Herrera-Malaver, B., Merlevede A,

758 Roncoroni M, Voordeckers K, Miraglia L., Teiling, C., Steffy, B., Taylor, M., Schwartz, A.,

759 Richardson, T., White, C., Baele, G., Maere, S., \& Verstrepen, K. J. (2016). Domestication

760 and divergence of Saccharomyces cerevisiae beer yeasts. Cell, 166(6), 1397-1410.

761 Ganesan, B., \& Weimer, B. C. (2017). Amino acid catabolism and its relationship to cheese

762 flavor outcomes. In P. L. H. McSweeney, P. F. Fox, P. D. Cotter, \& D. W. Everett (Eds.),

763 Cheese: Chemistry, Physics and Microbiology: Fourth Edition (Fourth Edi, Vol. 1, pp. 483-

764 516). Elsevier Ltd.

765 Gatto, L., \& Lilley, K. S. (2012). Msnbase-an R/Bioconductor package for isobaric tagged

766 mass spectrometry data visualization, processing and quantitation. Bioinformatics, 28(2),

767 288-289.

768 Gibbons, J. G., Salichos, L., Slot, J. C., Rinker, D. C., McGary, K. L., King, J. G., Klich

769 M.A., Tabb D.L., McDonald W.H. \& Rokas, A. (2012). The evolutionary imprint of

770 domestication on genome variation and function of the filamentous fungus Aspergillus

771 oryzae. Current Biology, 22(15), 1403-1409.

772

773

774

775

776

777

778

779

780

781

782

783

784

785

786

787
Gillot, G., Jany, J.-L., Coton, M., Le Floch, G., Debaets, S., Ropars, J., López-Villavicencio M., Dupont J., Branca A., Giraud T. \& Coton, E. (2015). (Fifty shades of blue $\square$ :) Insights into Penicillium roqueforti morphological and genetic diversity. PLoS ONE, 10(6), e0129849.

Gillot, G., Jany, J.-L., Poirier, E., Maillard, M., Debaets, S., Thierry, A., Coton E. \& Coton, M. (2017). Functional diversity within the Penicillium roqueforti species. International Journal of Food Microbiology, 241, 141-150.

Giraud, T., Koskella, B., \& Laine, A.-L. (2017). Introduction: microbial local adaptation: insights from natural populations, genomics and experimental evolution. Molecular Ecology, 26(7), 1703-1710.

Gladieux, P., Ropars, J., Badouin, H., Branca, A., Aguileta, G., De Vienne, D. M., Rodríguez De La Vega R.C., Branco S. \& Giraud, T. (2014). Fungal evolutionary genomics provides insight into the mechanisms of adaptive divergence in eukaryotes. Molecular Ecology, 23(4), $753-773$.

Gomri, G. (1946). Buffers in the range of pH 6.5 to 9.6. Proceedings of Society for Experimental Biology and Medicine, 6(1), 33-34. 
788

789

790

791

792

793

794

795

796

797

798

799

800

801

802

803

804

805

806

807

808

809

810

811

812

813

814

815

816

817

818

819

820

821

822

823

824

Gonçalves, M., Pontes, A., Almeida, P., Barbosa, R., Serra, M., Libkind, D., Hutzler M., Gonçalves P. \& Sampaio, J. P. (2016). Distinct domestication trajectories in top-fermenting beer yeasts and wine yeasts. Current Biology, 26(20), 2750-2761.

González de Llano, D., Ramos, M., Polo, C., Sanz, J., \& Martinez-Castro, I. (1990). Evolution of the volatile components of an artisanal blue cheese during ripening. Journal of Dairy Science, 73(7), 1676-1683.

González de Llano, D., Ramos, M., Rodriguez, A., Montilla, A., \& Juarez, M. (1992). Microbiological and physicochemical characteristics of Gamonedo blue cheese during ripening. International Dairy Journal, 2(2), 121-135.

Hébert, A., Forquin-Gomez, M. P., Roux, A., Aubert, J., Junot, C., Heilier, J. F., Landaud S., Bonnarmeb P. \& Beckerich, J. M. (2013). New insights into sulfur metabolism in yeasts as revealed by studies of Yarrowia lipolytica. Applied and Environmental Microbiology, 79(4), $1200-1211$.

ISO. (2004). Determination of water activity NF ISO 21807. International Standardisation Organisation. http://doi.org/ISO 21807:2004

Kinderlerer, J. L. (1993). Fungal strategies for detoxification of medium chain fatty acids. International Biodeterioration and Biodegradation, 32(1-3), 213-224.

Kinsella, J. E., \& Hwang, D. H. (1976). Enzymes of Penicillium roqueforti involved in the biosynthesis of cheese flavor. C R C Critical Reviews in Food Science and Nutrition, 8(2), 191-228.

Kopp, B., \& Rehm, H. J. (1979). Antimicrobial action of roquefortine. European Journal of Applied Microbiology and Biotechnology, 6, 397-401 (1979).

Kuhl, C., Tautenhahn, R., Böttcher, C., Larson, T. R., \& Neumann, S. (2012). CAMERA: An integrated strategy for compound spectra extraction and annotation of liquid chromatography/mass spectrometry data sets. Analytical Chemistry, 84(1), 283-289.

Labbe, M., \& Serres, J. P. (2004). Chroniques du Roquefort - De la préhistoire à l'aube industrielle. Grand Imprimeur, La Primaube, France.

Labbe, M., \& Serres, J. P. (2009). Chroniques du Roquefort: des Hommes, des entreprises, des marques, période moderne. Grand Imprimeur, La Primaube, France.

Larsen, M. D., \& Jensen, K. (1999). The effects of environmental conditions on the lipolytic activity of strains of Penicillium roqueforti. International Journal of Food Microbiology, 46(2), 159-166.

Larsen, M. D., Kristiansen, K. R., \& Hansen, T. K. (1998). Characterization of the proteolytic activity of starter cultures of Penicillium roqueforti for production of blue veined cheeses. International Journal of Food Microbiology, 43(3), 215-221.

Larson, G., \& Fuller, D. Q. (2014). The evolution of animal domestication. Annual Review of Ecology, Evolution, and Systematics, 45, 115-136. 
825

826

827

828

829

830

831

832

833

834

835

836

837

838

839

840

841

842

843

844

845

846

847

848

849

850

851

852

853

854

855

856

857

858

859

860

861

862

863

864
Lazuka, A., Auer, L., Bozonnet, S., Morgavi, D. P., O’Donohue, M., \& Hernandez-Raquet, G. (2015). Efficient anaerobic transformation of raw wheat straw by a robust cow rumenderived microbial consortium. Bioresource Technology, 196, 241-249.

Le Boucher, C., Courant, F., Jeanson, S., Chereau, S., Maillard, M., Royer, A. L., Thierry A., Dervilly-Pinel G., Le Bizec B. \& Lortal, S. (2013). First mass spectrometry metabolic fingerprinting of bacterial metabolism in a model cheese. Food Chemistry, 141(2), 10321040 .

Legras, J.-L., Galeote, V., Bigey, F., Camarasa, C., Marsit, S., Nidelet, T., Couloux A., Guy J., Franco-Duarte R. Marcet-Houben, M. Gabaldon, T., Schuller, D., Sampaio, J. P. \& Dequin, S. (2018). Adaptation of S. cerevisiae to fermented food environments reveals remarkable genome plasticity and the footprints of domestication. Molecular Biology and Evolution, 35(7), 1712-1727.

Libkind, D., Hittinger, C. T., Valeŕio, E., Gonçalves, C., Dover, J., Johnston, M., Gonçalves, P. \& Sampaio, J. P. (2011). Microbe domestication and the identification of the wild genetic stock of lager-brewing yeast. Proceedings of the National Academy of Sciences of the United States of America, 108(35), 14539-14544.

Liu, Z., Lozupone, C., Hamady, M., Bushman, F. D., \& Knight, R. (2007). Short pyrosequencing reads suffice for accurate microbial community analysis. Nucleic Acids Research, 35(18), e120.

Ludemann, V., Greco, M., Rodríguez, M. P., Basílico, J. C., \& Pardo, A. G. (2010). Conidial production by Penicillium nalgiovense for use as starter cultures in dry fermented sausages by solid state fermentation. LWT - Food Science and Technology, 43(2), 315-318.

Mahé, F., Rognes, T., Quince, C., de Vargas, C., \& Dunthorn, M. (2014). Swarm: robust and fast clustering method for amplicon-based studies. PeerJ, 2, e593.

Martín, J.-F., \& Coton, M. (2016). Blue cheese: microbiota and fungal metabolites. In J. Frias, C. Martinez-Villaluenga, \& E. Peñas (Eds.), Fermented Foods in Health and Disease Prevention (pp. 275-303). Elsevier Inc.

Mason, A. B., \& Dufour, J. P. (2000). Alcohol acetyltransferases and the significance of ester synthesis in yeast. Yeast, 16(14), 1287-1298.

Mayeux, J. V, Sandine, W. E., \& Elliker, P. R. (1962). A selective medium for detecting Leuconostoc organisms in mixed-strain starter cultures. Journal of Dairy Science, 45, 655656.

McSweeney, P. L. H. (1997). The flavour of milk and dairy products: III. Cheese: Taste. International Journal of Dairy Technology, 50(4), 123-128.

McSweeney, P. L. H., Fox, P. F., \& Ciocia, F. (2017). Metabolism of residual lactose and of lactate and citrate. In P. L. H. McSweeney, P. F. Fox, P. D. Cotter, \& D. W. Everett (Eds.), Cheese: Chemistry, Physics and Microbiology: Fourth Edition (Fourth Edi, pp. 411-421). Elsevier Ltd.

McSweeney, P. L. H., \& Sousa, M. J. (2000). Biochemical pathways for the production of flavour compounds in cheeses during ripening: A review. Le Lait, 80(3), 293-324. 
865

866

867

868

869

870

871

872

873

874

875

876

877

878

879

880

881

882

883

884

885

886

887

888

889

890

891

892

893

894

895

896

897

898

899

900

901

902

903

Moio, L., Piombino, P., \& Addeo, F. (2000). Odour-impact compounds of Gorgonzola cheese. Journal of Dairy Research, 67(2), 273-285.

Moreau, C. (1980). Le Penicillium roqueforti, morphologie, physiologie, intérêt en industrie fromagère, mycotoxines. Le Lait, 60(595-596), 254-271.

Mossel, D. A. A., Eelderink, I., Koopmans, M., \& Van Rossem, F. (1978). Optimisation of a MacConkey-type medium for the enumeration of Enterobacteriaceae. Laboratory Practices, 27, 1049-1050.

Mossel, D. A. A., Kleynen $\square$ Semmeling, A. M. C., Vincentie, H. M., Beerens, H., \& Catsaras, M. (1970). Oxytetracycline $\square$ Glucose $\square$ Yeast extract agar for selective enumeration of moulds and yeasts in foods and clinical material. Journal of Applied Bacteriology, 33(3), 454-457.

Nelson, F. E. (1940). The effect of the new standard milk agar on the plate count of dairy products. Journal of Bacteriology, 39(3), 263-272. Retrieved from https://www.ncbi.nlm.nih.gov/pmc/articles/PMC374570/

Oksanen, J., Blanchet, G. F., Firendly, M., Kindt, R., Legendre, P., McGlinn, D., Minchin, P.R., O’Hara, R.B., Simpson, G.L., Solymos, P., Stevens, M. H. H., Szoecs, E. \& Wagner, H. (2019). vegan: community ecology package. Retrieved from https://cran.rproject.org/package=vegan

Perrone, G., Samson, R. A., Frisvad, J. C., Susca, A., Gunde-Cimerman, N., Epifani, F., \& Houbraken, J. A. M. P. (2015). Penicillium salamii, a new species occurring during seasoning of dry-cured meat. International Journal of Food Microbiology, 193, 91-98.

Pham, V. T., Lacroix, C., Braegger, C. P., \& Chassard, C. (2016). Early colonization of functional groups of microbes in the infant gut. Environmental Microbiology, 18(7), 22462258 .

Pitt, J. I., \& Hocking, A. D. (2009). Fungi and food spoilage (Springer). Springer, Boston, MA.

Rognes, T., Flouri, T., Nichols, B., Quince, C., \& Mahé, F. (2016). VSEARCH: a versatile open source tool for metagenomics. PeerJ, 4, e2584.

Ropars J., Bennetot B., Caron T., Lo Y.C. and Giraud T. (2020a). The domestication of Penicillium cheese fungi. Comptes Rendus de l'Académie des Sciences - Biologie, 343, 155 176.

Ropars, J., Cruaud, C., Lacoste, S., \& Dupont, J. (2012). A taxonomic and ecological overview of cheese fungi. International Journal of Food Microbiology, 155(3), 199-210.

Ropars J., Didiot E., Rodriguez de la Vega R., Bennetot B., Coton M., Coton E., Snirc A., Le Prieur S. and Giraud T. (2020b). Domestication of the emblematic white cheese-making fungus Penicillium camemberti and its diversification into two varieties Current Biology, 30, 4441-4453.

Ropars, J., Lo, Y.-C., Dumas, É., Snirc, A., Begerow, D., Rollnik, T., Lacoste, S., Dupont, J., Giraud, T. \& López-Villavicencio, M. (2016). Fertility depression among cheese-making 
904 Penicillium roqueforti strains suggests degeneration during domestication. Evolution, 70(9),

905 2099-2109.

906 Ropars, J., López-Villavicencio, M., Dupont, J., Snirc, A., Gillot, G., Coton, M., Jany, J.-L., 907 Coton, E. \& Giraud, T. (2014). Induction of sexual reproduction and genetic diversity in the 908 cheese fungus Penicillium roqueforti. Evolutionary Applications, 7(4), 433-441.

909 Ropars, J., López-Villavicencio, M., Snirc, A., Lacoste, S., \& Giraud, T. (2017). Blue cheese910 making has shaped the population genetic structure of the mould Penicillium roqueforti.

911 PLOS ONE, 12(3), e0171387.

912 Ropars, J., Rodríguez de la Vega, R. C., Gouzy, J., Dupont, J., Swennen, D., Dumas, É., 913 Giraud, T. \& Branca, A. (2016). Diversity and mechanisms of genomic adaptation in 914 Penicillium. In R. P. de Vries, I. Benoit Gelber, \& A. Rordam (Eds.), Aspergillus and 915 Penicillium in the post-genomic era (HAL). Caister Academic Press.

916 Ropars, J., Rodríguez de la Vega, R. C., López-Villavicencio, M., Gouzy, J., Sallet, E., 917 Dumas, É., Lacoste, S., Debuchy, R., Dupont, J., Branca, A. \& Giraud, T. (2015). Adaptive 918 horizontal gene transfers between multiple cheese-associated fungi. Current Biology, 25(19), $9192562-2569$.

920 Rothe, M., Engst, W., \& Erhardt, V. (1982). Studies on characterization of blue cheese 921 flavour. Die Nahrung, 26(7/8), 591-602.

922 Roudot-Algaron, F. (1996). Le goût des acides aminés, des peptides et des protéines:

923 Exemple de peptides sapides dans les hydrolysats de caséines. Lait, 76(4), 313-348.

924 Schneider, C. A., Rasband, W. S., \& Eliceiri, K. W. (2012). NIH Image to ImageJ: 25 years 925 of image analysis. Nature Methods, 9(7), 671-675.

926 Schnurer, J. (1993). Comparison of methods for estimating the biomass of three food-borne 927 fungi with different growth patterns. Applied and Environmental Microbiology, 59(2), 552928555.

929 Searle, S. R., Speed, F. M., \& Milliken, G. A. (1980). Population marginal means in the 930 linear model: An alternative to least squares means. American Statistician, 34(4), 216-221.

931 Sicard, D., \& Legras, J.-L. (2011). Bread, beer and wine: Yeast domestication in the 932 Saccharomyces sensu stricto complex. Comptes Rendus - Biologies, 334(3), 229-236.

933 Smith, C. A., Want, E. J., O’Maille, G., Abagyan, R., \& Siuzdak, G. (2006). XCMS:

934 Processing mass spectrometry data for metabolite profiling using nonlinear peak alignment, 935 matching, and identification. Analytical Chemistry, 78(3), 779-787.

936 Snow, G. (2016). TeachingDemos: Demonstrations for teaching and learning. Retrieved from 937 https://cran.r-project.org/web/packages/TeachingDemos/index.html

938 Spinnler, H.-E. (2011). Rôle des lipides dans la perception olfactive des produits laitiers.

939 Sciences Des Aliments, 30, 103-120.

940 Terzaghi, B. E., \& Sandine, W. E. (1975). Improved medium for lactic streptococci and their 941 bacteriophages. Applied Microbiology, 29(6), 807-813. 
942 Thierry, A., Collins, Y. F., Abeijón Mukdsi, M. C., McSweeney, P. L. H., Wilkinson, M. G., 943 \& Spinnler, H.-E. (2017). Lipolysis and metabolism of fatty acids in cheese. In P. L. H.

944 McSweeney, P. F. Fox, P. D. Cotter, \& D. W. Everett (Eds.), Cheese: Chemistry, Physics and 945 Microbiology: Fourth Edition (Fourth Edi, pp. 423-444). Elsevier Ltd.

946 Urbanek, S., \& Horner, J. (2019). Cairo: R graphics device using Cairo graphics library for 947 creating high-quality bitmap (PNG, JPEG, TIFF), vector (PDF, SVG, PostScript) and display 948 (X11 and Win32) output. Retrieved from https://cran.r949 project.org/web/packages/Cairo/index.html

950 Vabre, S. (2015). Le sacre du Roquefort. Presses universitaires François Rabelais, Tours, 951 France.

952 Vallone, L., Giardini, A., \& Soncini, G. (2014). Secondary metabolites from Penicillium 953 roqueforti, a starter for the production of Gorgonzola cheese. Italian Journal of Food Safety, 954 3(3), 173-177 (2118).

955 Van Den Boogaart, K. G., Tolosana-Delgado, R., \& Bren, M. (2018). compositions: A

956 unified R package to analyze compositional data. Computers \& Geosciences, 34(4), 320-338.

957 Villanueva, R. A. M., Chen, Z. J., \& Wickham, H. (2016). ggplot2: Elegant graphics for data 958 analysis using the grammar of graphics (2nd Editio). Springer-Verlag New York.

959 Wickham, H. (2011). The split-apply-combine strategy for data analysis. Journal of 960 Statistical Software, 40(1), 1-29.

961 Wilke, C. O. (2019). cowplot: Streamlined plot theme and plot annotations for "ggplot2".

962 Retrieved from https://cran.r-project.org/web/packages/cowplot/index.html

963 Williams, A. G., Beattie, S. H., \& Banks, J. M. (2004). Enzymes involved in flavour 964 formation by bacteria isolated from the smear population of surface-ripened cheese.

965 International Journal of Dairy Technology, 57(1), 7-13.

966 Woo, A. H., \& Lindsay, R. C. (1984). Concentrations of major free fatty acids and flavor 967 development in Italian cheese varieties. Journal of Dairy Science, 67(5), 960-968. 


\section{Figure legends}

969

970 Figure 1: Experimental cheesemaking. (A) Experimental design for cheesemaking, using one

971 strain per cheese, and three different strains from each of the four Penicillium roqueforti

972 populations (non-Roquefort cheese in blue, Roquefort cheese in purple, silage/food spoiler in

973 orange, lumber/food spoiler in green, the lineages of which are shown on the left). Each assay

974 (February, March, April) corresponded to a single strain from each of the four populations, 975 with three production replicates at different times, different batches of unpasteurized milk 976 and with two cheeses produced per strain in each replicate. The identities of the strains used 977 are indicated on the left of each assay, for each of the four P. roqueforti populations. (B)

978 Photograph of the experimental cheeses after 20 days of maturation.

979

980 Figure 2: (A) Relative abundance of the six main bacterial operational taxonomic units in 981 cheeses made with strains from the four Penicillium roqueforti populations (non-Roquefort 982 cheese in blue, Roquefort cheese in purple, silage/food spoiler in orange and lumber/food 983 spoiler in green) in cheeses at 9 (left) and 20 (right) days of maturation. (B \& C) Mean

984 bacterial genus diversity; A: Shannon index, B: Inverse of Simpson index (= 1 - Simpson 985 index) for the operational taxonomic units detected by metabarcoding in 9-day cheeses (left) 986 and 20-day cheeses (right) made with strains from the four Penicillium roqueforti populations 987 (lumber/food spoiler in green, non-Roquefort cheese in blue, Roquefort cheese in purple and 988 silage/food spoiler in orange).

990 Figure 3: (A) Mean percentage blue area per cheese slice at 20, 90 and 180 days of 991 maturation, for cheeses made with strains from the four Penicillium roqueforti populations 992 (non-Roquefort cheese in blue, Roquefort cheese in purple, silage/food spoiler in orange and 993 lumber/food spoiler in green). Error bars indicate 95\% confidence intervals. (B) Illustration 
994 of the differences in mean percentage blue area per cheese slice at 180 days of maturation

995 between the four Penicillium roqueforti populations (non-Roquefort cheese in blue,

996 Roquefort cheese in purple, silage/food spoiler in orange and lumber/food spoiler in green).

997 Contrast and brightness have been standardized and the edges cropped.

998

999 Figure 4: Violin plot depicting the distribution of the sums of 3,864 non-targeted aqueous 1000 signal peak areas, weighted by their mass-to-charge ratios (“m/z"), obtained in positive 1001 ionization mode for 90-day cheeses made with strains from the four Penicillium roqueforti 1002 populations (lumber/food spoiler in green, non-Roquefort cheese in blue, Roquefort cheese in 1003 purple and silage/food spoiler in orange). Boxplots within violin plots represent the median 1004 (center line), the 25th and 75th percentiles (box bounds), the 5th and 95th percentiles 1005 (whiskers), the points being the outliers from these 95th and 5th percentiles. The red dots 1006 depict the mean values.

1007

1008 Figure 5: Violin plot depicting the distribution of the sums of 3,094 non-targeted organic 1009 signal peak areas, weighted by their mass-to-charge ratios (“m/z"), obtained in negative ionization mode for 90-day cheeses made with strains from the four Penicillium roqueforti

1011 populations (lumber/food spoiler in green, non-Roquefort cheese in blue, Roquefort cheese in 1012 purple and silage/food spoiler in orange). Boxplots within violin plots represent the median 1013 (center line), the 25th and 75th percentiles (box bounds), the 5th and 95th percentiles 1014 (whiskers), and the red dots depict the mean values.

1016 Figure 6: Volatile compound production (integrated peak areas from chromatograms in 1017 arbitrary units) in 90-day cheeses inoculated with strains from the four Penicillium roqueforti 1018 populations (non-Roquefort cheese in blue, Roquefort cheese in purple, silage/food spoiler in 
1019

1020

1021

1022

1023

1024

1025

1026

1027

1028

1029

1030

1031

1032

1033

1034

1035

1036

1037

1038

1039

1040

1041 binarization with a grayscale of 102 on the red channel. White and black correspond to pixel 1042 classification: in white, the inner part of the cheese and empty cavities; in black, cavities 1043 filled with the fungus.

orange and lumber/food spoiler in green). The areas for each family of compounds are the sum of the integrated areas of the compounds belonging to the family concerned. Alcohols I and II are derived from proteolysis and lipolysis, respectively. Acids I, II and III are derived from proteolysis, glycolysis and lipolysis, respectively (Supplementary Table 3). The color of the titles indicates the affiliation of the compounds to their families, as in Figure S6.

Figure 7: Integrated surface area (from chromatograms in arbitrary units) of the three most discriminant methyl ketones (A) and four secondary alcohols (B) for each assay (February, March, April) for the three strains of each Penicillium roqueforti population (lumber/food spoiler in green, non-Roquefort cheese in blue, Roquefort cheese in purple, silage/food spoiler in orange). Error bars represent standard deviations across cheese replicates.

\section{Figure legends for the Supplementary Material}

Figure S1: Abundance of microorganisms (in log colony-forming units/g) of the eight types monitored at various stages of cheese maturation (i.e. unpasteurized milk, 9, 20, 90 and 180 days), for each of the four Penicillium roqueforti populations used to inoculate the cheeses (non-Roquefort cheese in blue, Roquefort cheese in purple, silage/food spoiler in orange and lumber/food spoiler in green). Error bars represent standard deviations across assays.

Figure S2: Illustration of image processing for estimation of the percentage blue area on cheese slices: (a) example of an unprocessed image of a cheese slice; (b) image after brightness and contrast standardization; (c) image after cropping; (d) corresponding image 
1045 Figure S3: Differences in aminoacid content between cheeses according to the population-oforigin of the Penicillium roqueforti strains. A. Discrimination between 90-day cheeses made with cheese (blue) and non-cheese (green) P. roqueforti populations (left), or Roquefort

1048 cheese (purple) and non-Roquefort cheese (blue) P. roqueforti populations (right), based on 1049 the abundance of the 23 identified amino acids present, according to an orthogonal signal1050 corrected partial least squares (PLS) discriminant analysis. Vertical and horizontal axes 1051 represent PLS1 and PLS 2 scores and gray arrows represent the relative contribution of 1052 loadings of signals significantly discriminating the group considered in a $t$-test with jackknife resampling. B. Abundance of molecules from particular classes detected in cheeses: mean integrated peak area from chromatograms in arbitrary units (bars, left axis) and cumulative 1055 percentage (line with dots, right axis) of aqueous extracts across all 90-day cheeses.

1057 Figure S4: Violin plot depicting the distribution of the sums of 8,472 non-targeted organic signal peak areas, weighted by their mass-to-charge ratios ("m/z"), obtained in positive ionization mode for 90-day cheeses made with strains from the four Penicillium roqueforti populations (lumber/food spoiler in green, non-Roquefort cheese in blue, Roquefort cheese in purple and silage/food spoiler in orange). Boxplots within violin plots represent the median 1062 (center line), the 25th and 75th percentiles (box bounds), the 5th and 95th percentiles 1063 (whiskers), and the red dots depict the mean values.

1064

1065 Figure S5: Non-protein nitrogen levels at 20, 90 and 180 days of maturation, and water 1066 activity at 90 and 180 days of maturation. Comparison of cheeses made with strains from 1067 different Penicillium roqueforti populations (non-Roquefort cheese in blue, Roquefort cheese 1068 in purple, silage/food spoiler in orange and lumber/food spoiler in green). Error bars indicate 
$106995 \%$ confidence intervals.

1070

1071 Figure S6: Discrimination between 90-day cheeses inoculated with strains from the four 1072 Penicillium roqueforti populations (non-Roquefort cheese in blue, Roquefort cheese in 1073 purple, silage/food spoiler in yellow and lumber/food spoiler in green), based on the 1074 abundance of 41 volatile compounds in an orthogonal signal-corrected partial least squares 1075 (PLS) discriminant analysis. Vertical and horizontal axes represent the PLS1 and PLS2 1076 variances, and arrows represent the relative contributions of compound odor loadings 1077 significantly discriminating the group considered (according to 1078 www.thegoodscentscompany.com) in a $t$-test with jackknife resampling. The odor colors 1079 indicate the families in Figure 6 to which the associated compounds belong.

1081 Conflict of interest: TC, MLP, SB, DR and MP were employed by SAS LIP, which 1082 produces starters for fermented food products, during the course of the study and therefore 1083 declare a competing financial interest. None of the other authors has any conflict of interest to 1084 declare. With the exception of TC employed by the LIP, the funders had no role in decisions 1085 concerning design, data analysis and interpretation, or in the decision to submit the work for 1086 publication. 
Figure 1

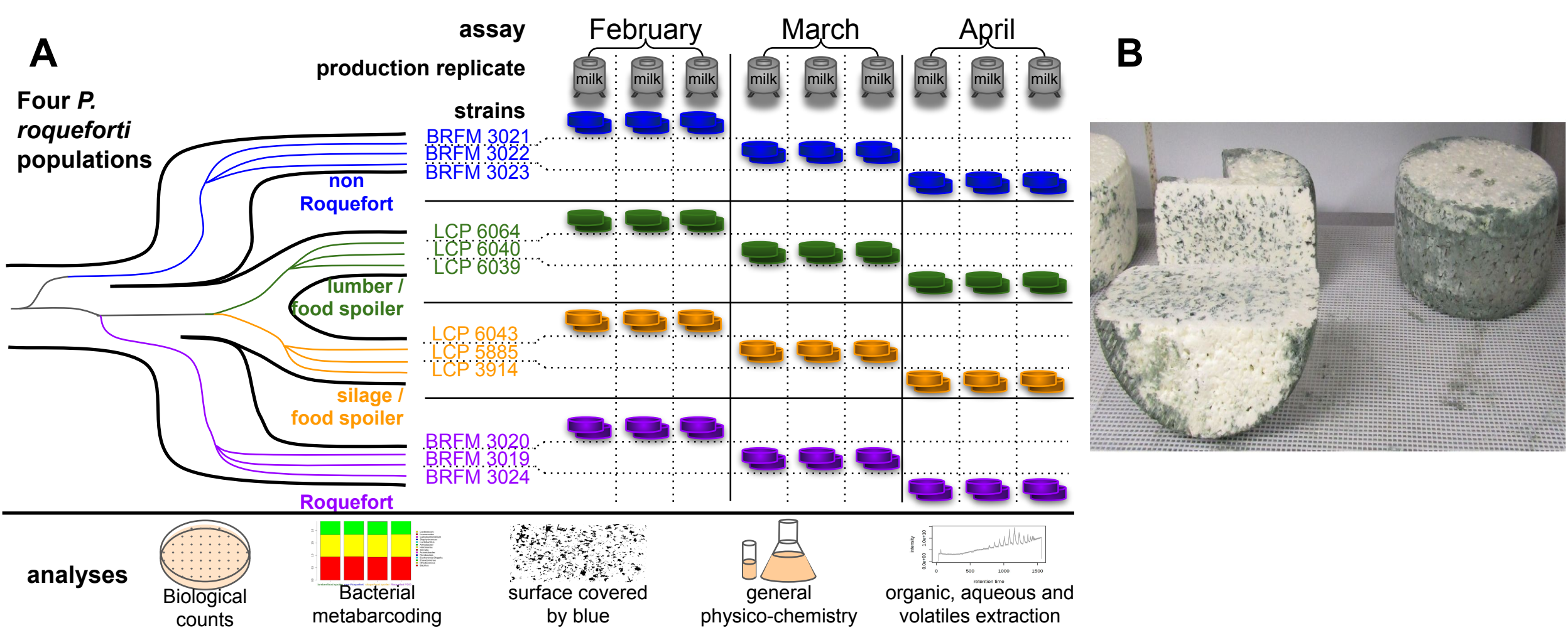


Figure 2A
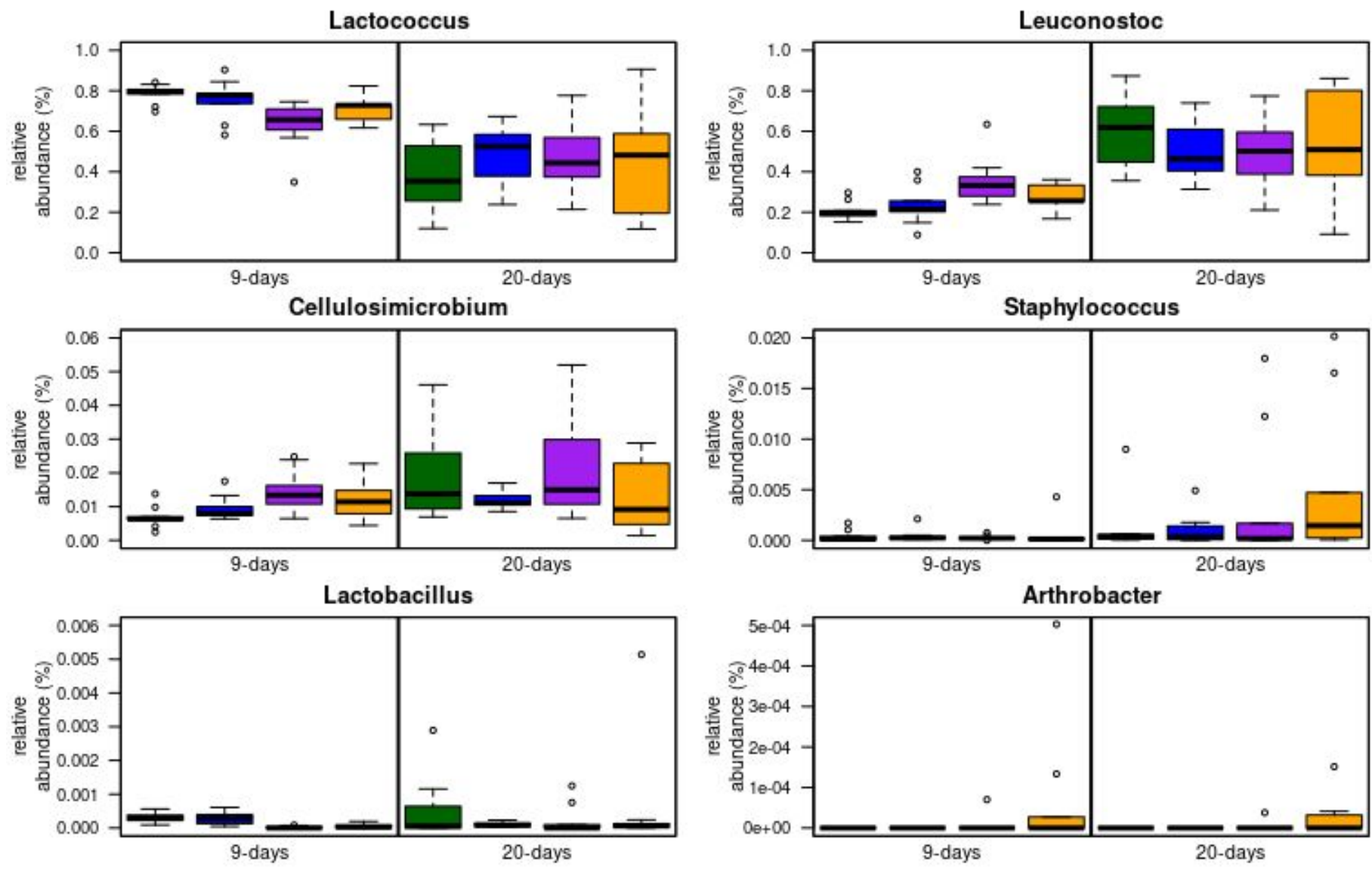

Penicillium roqueforti population

- lumber/food spoiler

non-Roquefort

- Roquefort

ㅁ silage/food spoiler 
Figure 2B

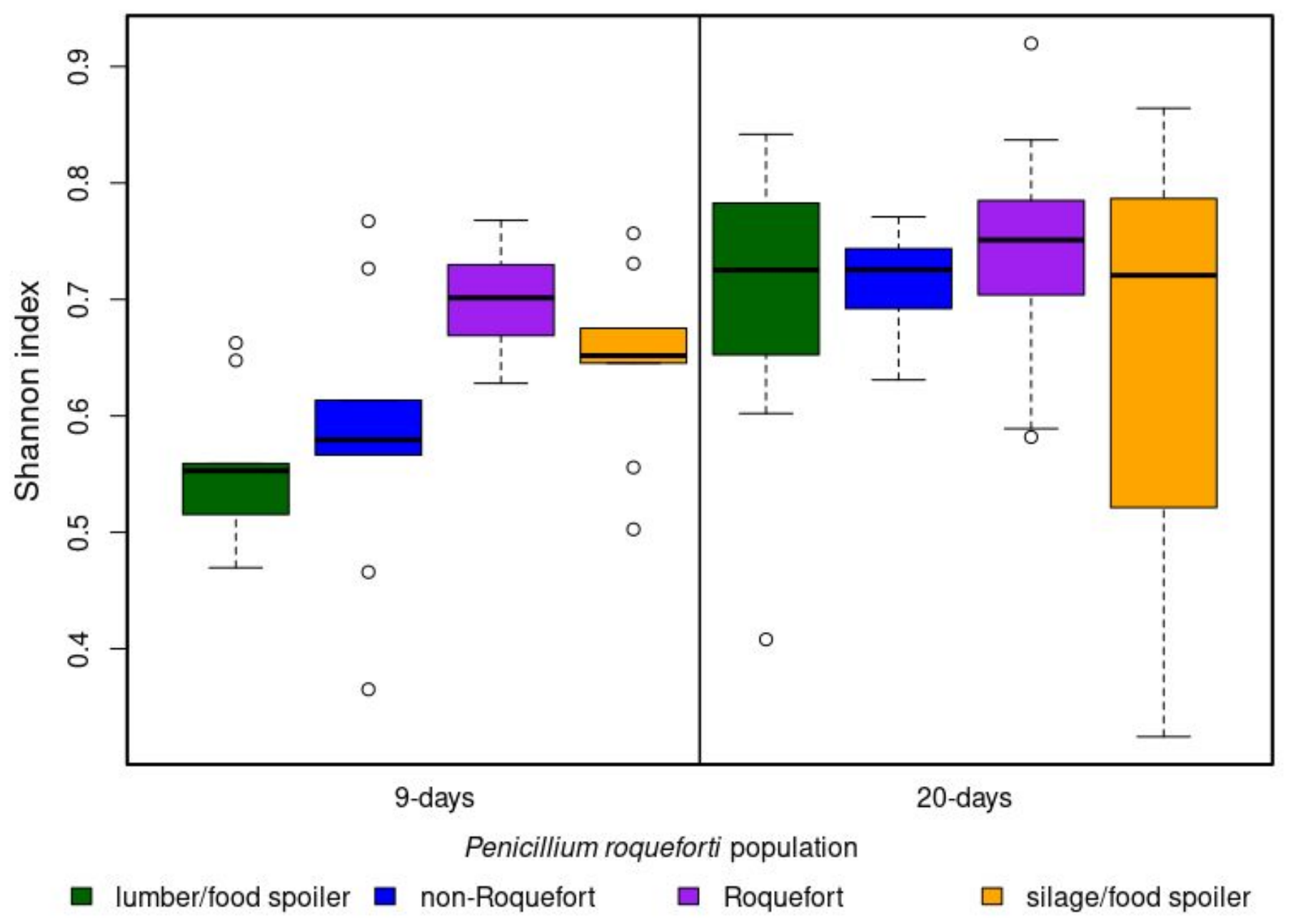


Figure 2C

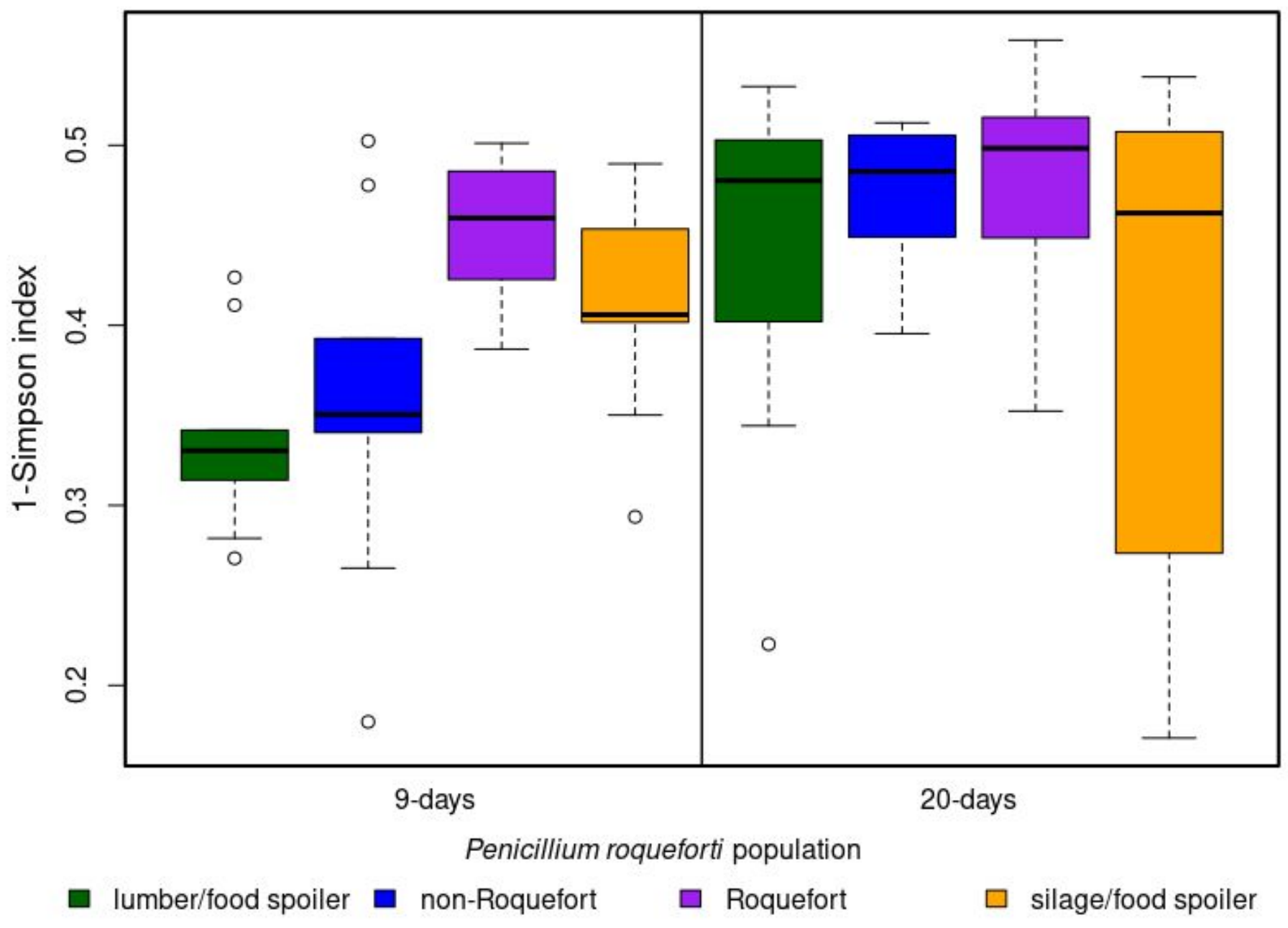


Figure $3 \mathrm{~A}$

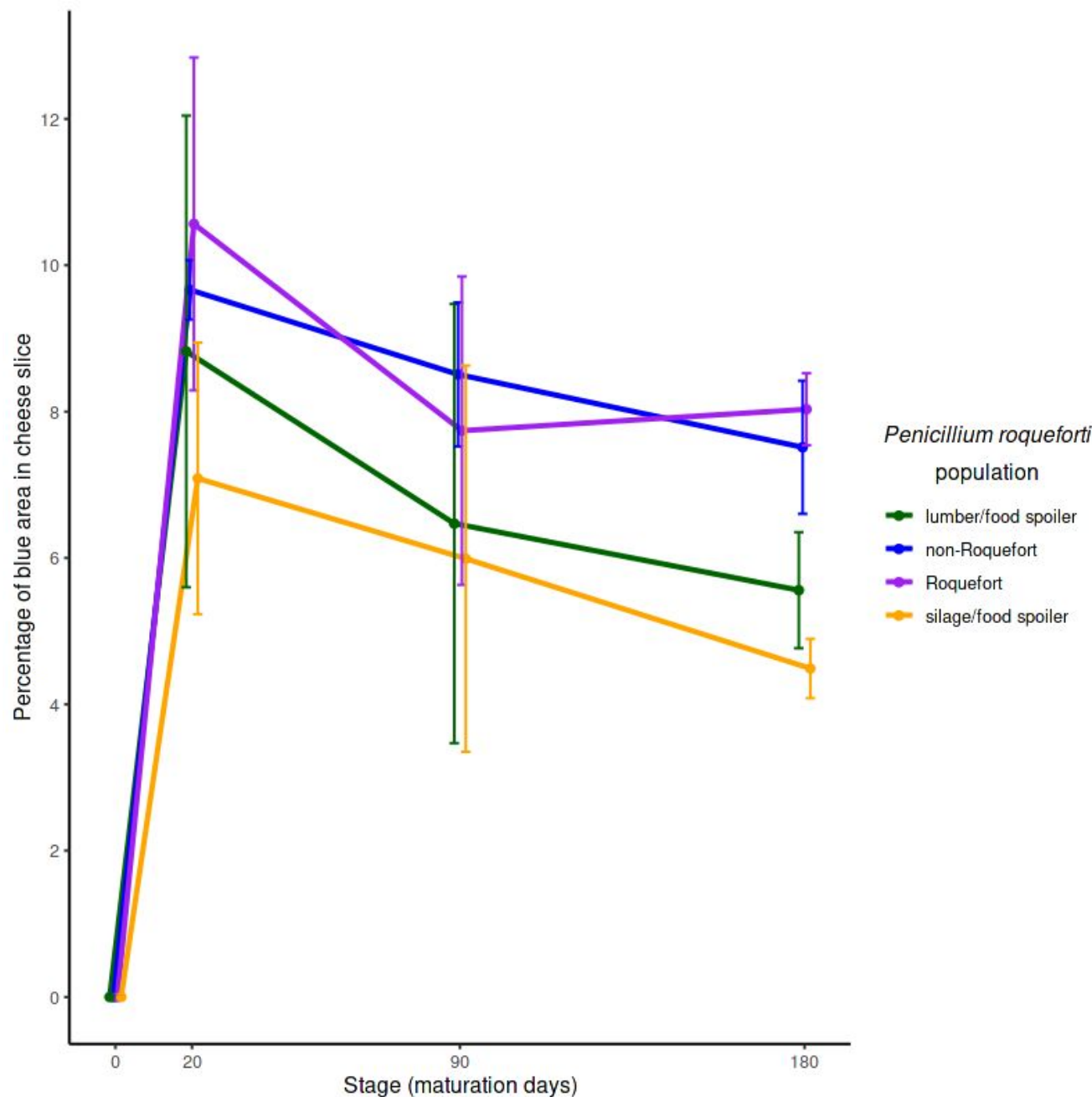


Figure 3B

lumber/food spoiler (5.2\%)

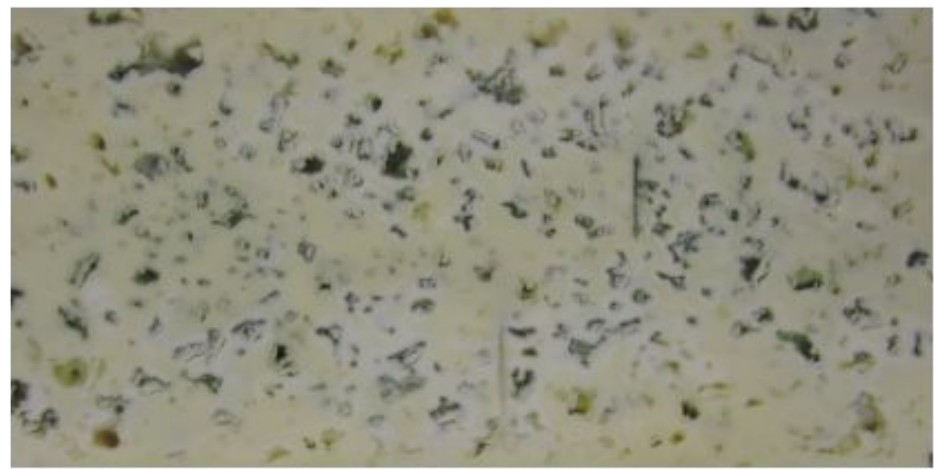

silage/food spoiler (5.5\%)

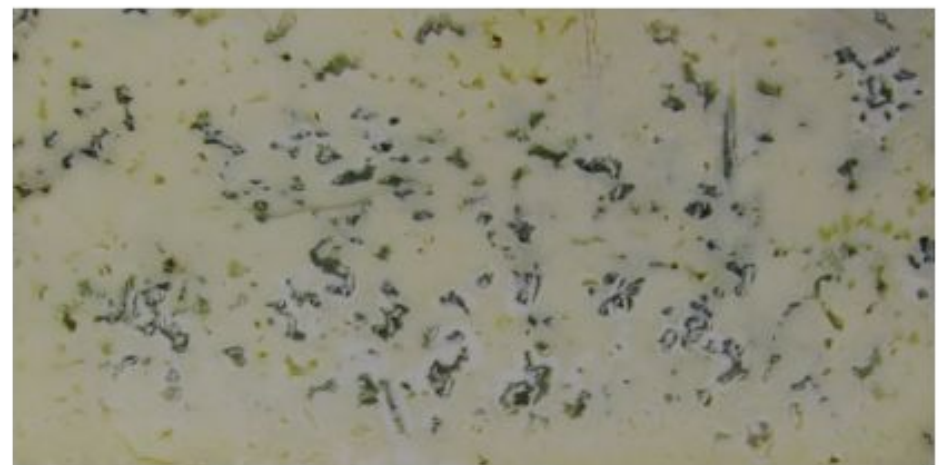

non-Roquefort (10.2\%)

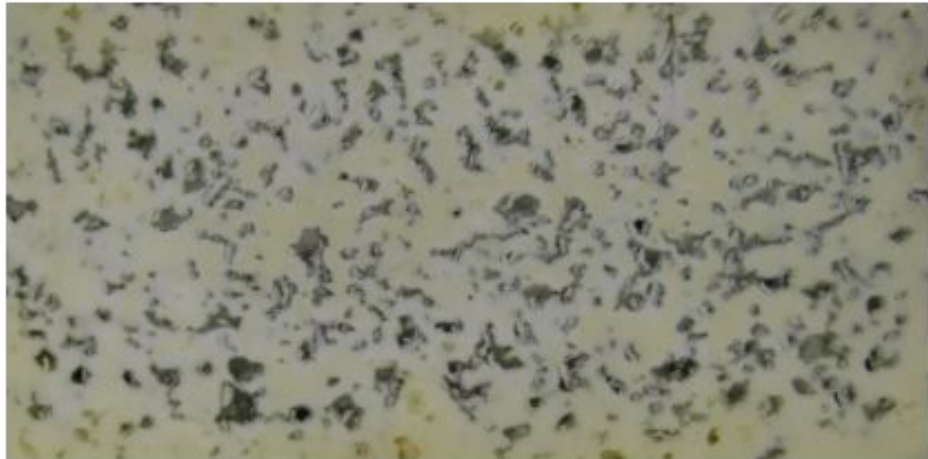

Roquefort (11.8\%)

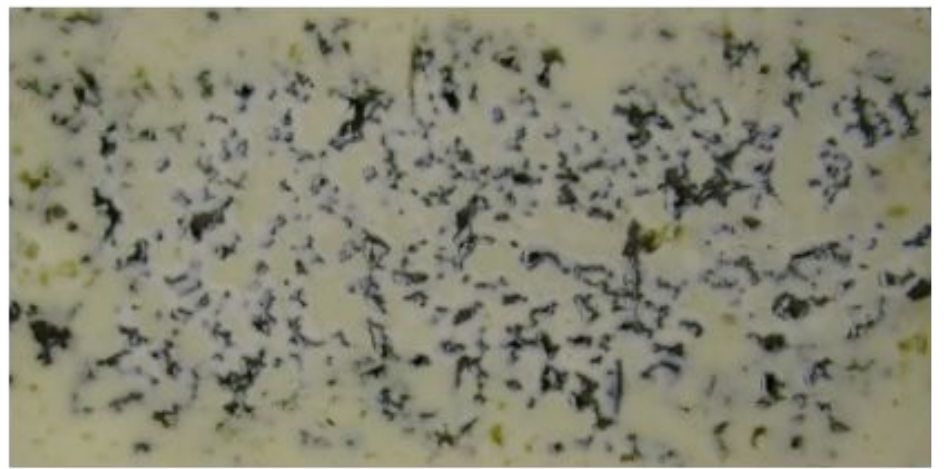




$$
\text { If } f^{\prime}=
$$


Figure 5

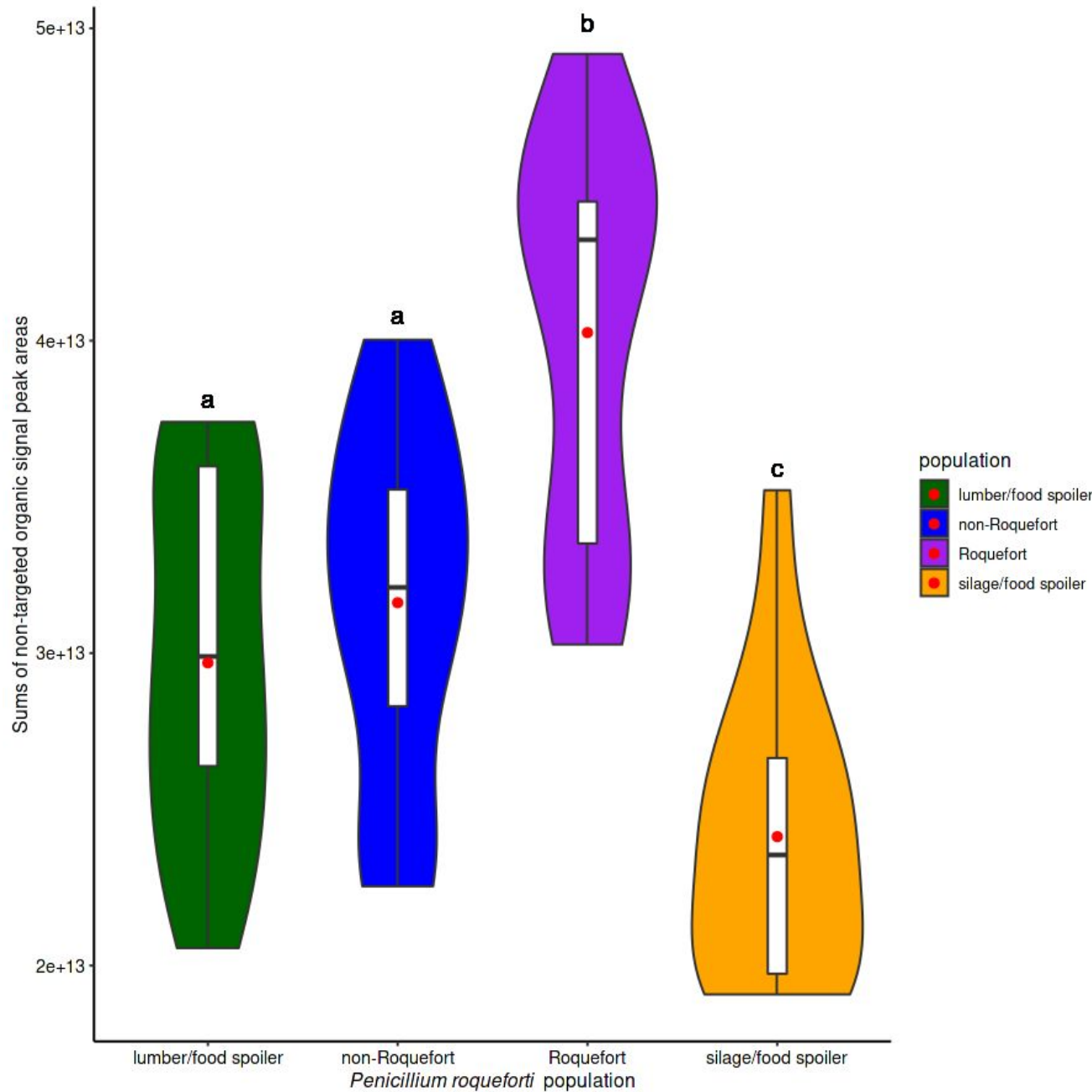




\section{Figure 6}
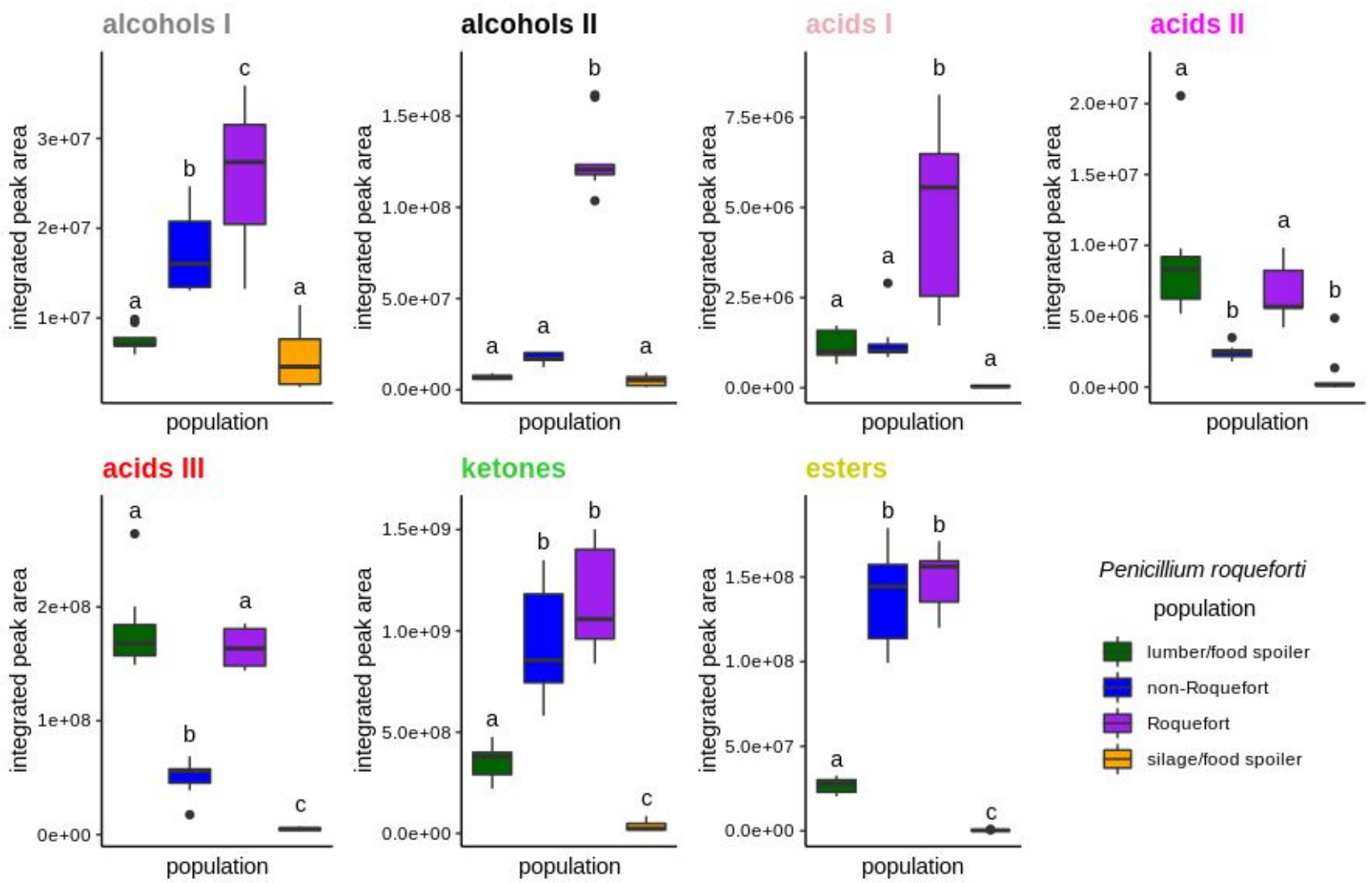

Penicillium roqueforti population

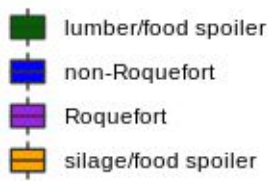


